\title{
Effect of surface contamination on interfacial mass transfer rate
}

\author{
J. G. WIS I I N K ${ }^{1} \dagger ， H . H E R L I N A^{2} ， Y . A K A R^{1}$ \\ AND M. UHL M A N N2
}

\author{
${ }^{1}$ Department of Mechanical, Aerospace and Civil Engineering, Brunel University London, \\ Kingston Lane, Uxbridge, UB8 3PH, UK \\ ${ }^{2}$ Institute for Hydromechanics, Karlsruhe Institute of Technology, Kaiserstr.12, \\ 76131 Karlsruhe, Germany
}

(Received ?; revised ?; accepted ?. - To be entered by editorial office)

The influence of surface contamination upon the mass transfer rate of a low diffusivity gas across a flat surface is studied using direct numerical simulations. The interfacial mass transfer is driven by isotropic turbulence diffusing from below. Similar to Shen et al. ( $J$. Fluid Mech. 506, 2004, pp. 79-115) the surface contamination is modelled by relating the normal gradient of the horizontal velocities at the top to the horizontal gradients of the surfactant concentrations. A broad range of contamination levels is considered, including clean to severely contaminated conditions. The time-averaged results show a strong correlation between the gas transfer velocity and the clean surface fraction of the surface area. In the presence of surface contamination the mass transfer velocity $K_{L}$ is found to scale as a power of the Schmidt number, i.e. $S c^{-q}$, where $q$ smoothly transitions from $q=1 / 2$ for clean surfaces to $q=2 / 3$ for very dirty interfaces. A power law $K_{L} \propto S c^{-q}$ is proposed in which both the exponent $q$ and the constant of proportionality become functions of the clean surface fraction.

\section{Key words:}

\section{Introduction}

The direct numerical simulations (DNS) presented in this paper are carried out in order to obtain a detailed understanding of the effect of various levels of contamination on gas transfer across the air-water interface driven by isotropic turbulence diffusing from below. Because of their low mass diffusivity (high Schmidt number) in water, the gas transfer process for low to moderate soluble gases (e.g. oxygen, carbon dioxide, carbon monoxide, methane) is controlled by the hydrodynamics at the liquid side (e.g. Jähne \& Haussecker 1998) which significantly depend on the surface condition. In nature, a truly clean surface is almost impossible to maintain. Hence, it is of key importance to understand in detail the effects of surface contamination on the interfacial mass transfer. In the natural environment, several types of surface contamination can be found. In the present paper we assume the surfactant to be insoluble, such as oleyl alcohol. However, the dynamics of low-soluble monolayer type surfactants with a significantly higher concentration at the surface than in the bulk can be approximately simulated using the aforementioned assumption. Examples of such monolayer films due to organic matter can be found in e.g. Espedal et al. (1996). Other types of contamination, such as the presence of a thick

$\dagger$ Email address for correspondence: jan.wissink@brunel.ac.uk 
layer of crude oil on the water surface, fall outside the scope of this paper. Because of the different mechanisms of scalar transfer, to simulate this type of contamination a two layer structure with different molecular diffusivities needs to be resolved.

Contamination by e.g. surfactants changes the near surface hydrodynamic conditions by the generation of tangential stresses due to variations in surface elasticity, which under clean conditions is constant. These stresses damp the turbulent eddies near the surface (see Davies 1972). For example, Handler et al. (2003) and Shen et al. (2004) studied the effect of surfactants on free-surface turbulent flow and showed that the surface divergence and associated upwellings and downwellings can be significantly reduced by the presence of even small amounts of surfactants. For large amounts of surfactants Shen et al. (2004) showed that the surface divergence becomes negligibly small and, hence, these up and downwellings almost completely disappear. Consequently, this damping of vertical turbulent motion close to the surface will lead to a decrease in the transfer velocity, $K_{L}$, of atmospheric gases across the air-water interface. For instance, in the experiments of Asher \& Pankow (1986) and McKenna \& McGillis (2004), compared to clean conditions, surface contamination was found to result in a reduction in $K_{L}$ of up to $80 \%$. This is in agreement with the DNS results of Herlina \& Wissink (2016) who studied the limit case of severe contamination (i.e. a no-slip condition) at a Schmidt number of $S c=500$, which is typical for oxygen. In the hybrid DNS/LES study of Hasegawa \& Kasagi (2008) the effect of surface contamination on surface shear driven interfacial mass transfer was studied for $S c=1$ and 100. For the higher value of $S c$ a reduction in $K_{L}$ of about $65 \%$ compared to clean conditions was obtained.

The transfer of atmospheric gases into water is dominated by molecular diffusion resulting in a concentration boundary layer adjacent to the surface. The thickness of this boundary layer (typically $10-1000 \mu \mathrm{m}$ for $S c \approx 500-700$ ) is usually controlled by turbulence generated by e.g. wind-shear, buoyancy, or bottom-shear. Near the surface this turbulent motion periodically brings up unsaturated fluid from the bulk. During a certain time-interval $\Delta t$ (the renewal time) this fluid is subsequently transported along the surface, where it becomes saturated with atmospheric gases due to diffusion, before it is returned to the bulk. The surface renewal model of Danckwerts (1951) uses an exponential distribution of the renewal time to obtain the relationship $K_{L} \sim \sqrt{D r}$, where $D$ is the diffusion coefficient and $r=1 / \Delta t$ is the surface renewal rate. In this surface renewal model $r$ implicitly describes the hydrodynamical effects and needs to be determined experimentally. Using DNS results of open-channel flow, Kermani et al. (2011) showed that Danckwerts' assumption of a constant surface renewal rate is only approximately valid for larger (older) surface ages.

Fortescue \& Pearson (1967) assumed that the largest eddies in the flow determine the surface renewal rate so that $r$ is estimated by $u_{\infty} / \Lambda$, where $u_{\infty}$ is the root-mean-square (rms) of the turbulent fluctuations and $\Lambda$ is the characteristic length scale of the largest eddies. Hence, the transfer velocity predicted by using this "large-eddy model" is defined by $K_{L} \propto \sqrt{D u_{\infty} / \Lambda}$. Alternatively, by assuming that small rather than large eddies determine the surface renewal rate, Banerjee et al. (1968) and Lamont \& Scott (1970) derived the "small-eddy model", where $r$ is approximated by $(\epsilon / \nu)^{1 / 2}$, in which $\epsilon$ is the turbulent dissipation rate near the surface and $\nu$ is the kinematic viscosity. The transfer velocity is subsequently estimated by $K_{L} \propto \sqrt{D(\epsilon / \nu)^{1 / 2}}$. By non-dimensionalising the large and small eddy models using $u_{\infty}$ and $\Lambda$, we obtain

$$
\frac{K_{L}}{u_{\infty}}=c_{1} S c^{-1 / 2} R_{T}^{-1 / 2}
$$


and

$$
\frac{K_{L}}{u_{\infty}}=c_{2} S c^{-1 / 2} R_{T}^{-1 / 4},
$$

respectively, where $S c=\nu / D, R_{T}=u_{\infty} \Lambda / \nu$ is the turbulent Reynolds number and $c_{1}$, $c_{2}$ are constants of proportionality. Note that in $(1.2) \epsilon$ is estimated by $\epsilon=u_{\infty}^{3} / \Lambda$. In this form, the only difference between the two models lies in the exponent of $R_{T}$. Theofanous et al. (1976) recognised the existence of two regimes, where the large and small eddy models are valid for low and high $R_{T}$, respectively, with a critical turbulent Reynolds number of $R_{T, \text { crit }} \approx 500$.

An important variant of the surface renewal model is the surface divergence model of McCready et al. (1986),

$$
K_{L}=c_{\beta} \sqrt{D \beta_{r m s}},
$$

where the rms of the surface divergence, $\beta_{r m s}$, is used as a measure for the renewal rate. They showed the importance of surface divergence in interfacial mass transfer. This result was subsequently confirmed in various experimental and numerical studies (e.g. McKenna \& McGillis 2004; Turney 2016; Magnaudet \& Calmet 2006; Kermani et al. 2011; Herlina \& Wissink 2014; Wissink \& Herlina 2016) showing that surface divergence can provide a good quality prediction of the transfer velocity, though the constant of proportionality $c_{\beta}$ was found to vary significantly from case to case. To circumvent uncertainties in the definition of a renewal event, some researchers proposed mixed models of surface renewal and divergence (e.g. Peirson \& Banner 2003).

In the numerical investigations of Shen et al. (2004), Hasegawa \& Kasagi (2008) and Khakpour et al. (2011) a drastic reduction in $\beta_{r m s}$ was observed with increasing contamination levels. Based on this it was concluded that using a no-slip interfacial boundary condition would be a good approximation of a severely contaminated air-water interface. Based on the findings of Ledwell (1984) and Coantic (1986), it is now generally accepted that for a free-slip surface (clean conditions) the transfer velocity $K_{L}$ scales with $S c^{-1 / 2}$, while for a no-slip surface (severely contaminated conditions) $K_{L}$ scales with $\mathrm{Sc}^{-2 / 3}$. This is in agreement with e.g. Hasegawa \& Kasagi (2008) who observed that for higher levels of contamination the interfacial mass transfer scaling as a power of the Schmidt number "switches" from $S c^{-0.5}$ to $S c^{-0.7}$ for clean and severely contaminated surfaces, respectively .

For such a no-slip boundary condition Herlina \& Wissink (2016) proposed a modified version of the dual-regime model of Theofanous et al. (1976) given by

$$
\frac{K_{L}}{u_{\infty}} \propto S c^{-2 / 3} R_{T}^{-1 / 2} \text { for } \quad R_{T}<R_{T, c r i t},
$$

and

$$
\frac{K_{L}}{u_{\infty}} \propto S c^{-2 / 3} R_{T}^{-1 / 4} \text { for } \quad R_{T}>R_{T, c r i t} .
$$

For isotropic turbulence driven mass transfer, the validity of both the large (1.4) and small (1.5) eddy variant for the no-slip boundary condition was subsequently verified by Herlina \& Wissink (2016) using DNS data for both low $R_{T}$ (up to $S c=500$ ) and high $R_{T}$ (up to $S c=32$ ). For a free-slip boundary condition (clean surface condition), on the other hand, the validity of the scaling of $K_{L}$ in (1.1) was verified in Herlina \& Wissink (2014) for $S c$ up to 500 and turbulent Reynolds numbers up to $R_{T} \approx 500$. Also, for buoyancy driven mass transfer Wissink \& Herlina (2016) obtained the correct scaling behaviour of $K_{L}$ with $S c$ for $R_{T} \leqslant 50$ and $S c$ up to 500 .

It remains to be determined what scaling would apply (if any) for small to moderate 
levels of contamination. To investigate this let us assume that for any surface condition $K_{L}$ can be approximated by

$$
\frac{K_{L}}{u_{\infty}}=c S c^{-q} R_{T}^{-r}
$$

where $r=1 / 2$ and $1 / 4$ for the low and high $R_{T}$ regimes, respectively. In this expression the power $q$ is related to the level of contamination at the surface. Note that for other flow regimes the scalings in (1.1), (1.2), (1.4) and (1.5) might not be valid, as evidenced by the large spread of parameters obtained in previous works (e.g. Notter \& Sleicher 1971; Na \& Hanratty 2000; Jähne \& Haussecker 1998). Therefore, we decided to avoid any bias due to mean shear and use isotropic turbulence when studying the effect of various levels of surface contamination on interfacial gas transfer.

As in our previous DNS calculations, surface waves are assumed to be very shallow so that the interface can be modelled using a rigid lid assumption. The contamination level is characterised by $M a / C a_{T}$, where $M a$ is the Marangoni number and $C a_{T}$ is the turbulent capillary number (details in $\S 2.3$ ). In the results presented below, it will be shown that for small levels of contamination, parts of the surface area become virtually surfactant-free. A model is subsequently proposed and verified that predicts $K_{L}$ using the surfactant-free fraction of the surface area.

\section{Numerical aspects}

\subsection{Governing equations}

The problem that is simulated concerns the reduction in interfacial gas transfer due to surface contaminations in a turbulent water environment driven by isotropic turbulence diffusing from below. The dimensionless incompressible Navier-Stokes equations are defined by the continuity equation

and the momentum equations

$$
\frac{\partial u_{i}}{\partial x_{i}}=0
$$

$$
\frac{\partial u_{i}}{\partial t}+\frac{\partial u_{i} u_{j}}{\partial x_{j}}=-\frac{\partial p}{\partial x_{i}}+\frac{1}{R e} \frac{\partial^{2} u_{i}}{\partial x_{j} \partial x_{j}} \quad(i=1,2,3),
$$

where $x_{1}=x, x_{2}=y$ are the horizontal (surface-parallel) directions and $x_{3}=z$ is the vertical (surface-normal) direction, $u_{1}=u, u_{2}=v$ and $u_{3}=w$ are the velocities in the $x, y$, and $z$ directions, respectively, $p$ is the pressure, $R e$ is the Reynolds number and $t$ denotes time.

The dimensionless scalar concentration $c^{*}$ is governed by the following advection diffusion equation

where

$$
\frac{\partial c^{*}}{\partial t}+\frac{\partial u_{j} c^{*}}{\partial x_{j}}=\frac{1}{R e S c} \frac{\partial^{2} c^{*}}{\partial x_{j} \partial x_{j}} \quad(j=1,2,3),
$$

$$
c^{*}=\frac{c-c_{b, 0}}{c_{s}-c_{b, 0}},
$$

in which $c_{b, 0}$ is the initial concentration in the bulk and $c_{s}$ is the concentration at the surface, which is assumed to be fully saturated $\left(c_{s}=1\right)$ at all times. At the bottom of the domain a Neumann boundary condition of zero scalar flux $(\partial c / \partial z=0)$ is imposed.

At the surface the following two-dimensional transport equation for the surfactant concentration is solved

$$
\frac{\partial \gamma^{*}}{\partial t}+\frac{\partial u_{j} \gamma^{*}}{\partial x_{j}}=\frac{1}{R e S c_{s}} \frac{\partial^{2} \gamma^{*}}{\partial x_{j} \partial x_{j}} \quad(j=1,2),
$$


where $\gamma^{*}$ is defined as the surfactant concentration, $\gamma$, non-dimensionalised by the equilibrium concentration $\gamma_{0}$. Note that a rigid lid assumption was employed and the surfactant concentration at the surface was assumed to be conserved at all times. The latter implies that the surfactant is insoluble, whereby absorption and desorption processes as well as chemical kinetics that may affect the surfactant concentration are ignored. An example of such a surfactant often used in experiments is oleyl alcohol (e.g. McKenna \& McGillis 2004; Salter et al. 2011). Typical concentrations of this surfactant that would correspond to the levels of contamination investigated in this paper range from 0 to about $0.1 \mu \mathrm{g} / \mathrm{cm}^{2}$.

Similar to $\gamma^{*}$, the surface tension $\sigma$ is also made dimensionless using its equilibrium value $\sigma_{0}$. At the water surface $\sigma^{*}=\sigma / \sigma_{0}$ is assumed to linearly depend on $\gamma^{*}$ - which is a significant idealisation of the real-world behaviour of surfactants - so that the Marangoni number, defined by

$$
M a=-\left.\frac{d \sigma^{*}}{d \gamma^{*}}\right|_{\gamma^{*}=1},
$$

is constant. Note that it is assumed that the surface tension only depends on the surfactant concentration, other factors (such as temperature variations and interfacial viscosity) are assumed to be negligible. Also, in the remainder of this paper $c$ and $\gamma$ rather than $c^{*}$ and $\gamma^{*}$, respectively, will be used to refer to the non-dimensional concentrations.

Based on the model presented in Shen et al. (2004) the effect of the surface contamination on the near surface velocity fluctuations is modeled by relating the normal gradient of the horizontal velocities at the surface $\left(z=L_{z}\right)$ to the horizontal gradients of the surfactant concentration

$$
\begin{aligned}
& \left.\frac{\partial u}{\partial z}\right|_{z=L_{z}}=-\frac{M a}{C a} \frac{\partial \gamma}{\partial x} \\
& \left.\frac{\partial v}{\partial z}\right|_{z=L_{z}}=-\frac{M a}{C a} \frac{\partial \gamma}{\partial y}
\end{aligned}
$$

where $C a=\mu U / \sigma$ is the capillary number and $\mu$ is the dynamic viscosity. In the presence of mean shear, the parameter $\mathrm{Ma} / \mathrm{Ca}$ is typically presented using the equivalent expression $R e M a / W e$, where $W e$ is the Weber number. Because in our case the mean shear is zero, we prefer to use the capillary number. Note that when slight vertical deformations of the interface are simulated, a more sophisticated model is required (e.g. Tsai 1996).

\subsection{Numerical method}

The incompressible Navier-Stokes equations were discretised using fourth-order-accurate central discretisations of advection and diffusion (see Wissink 2004) combined with the second-order Adams-Bashforth method for the time integration. The scalar transport equations (2.3) and (2.5) were solved using the fifth-order accurate WENO scheme of Liu et al. (1994) for the convective terms combined with a fourth-order accurate central discretisation for the diffusion. For the time-integration of the scalar equations a threestage Runge-Kutta method was used. To deal with low scalar diffusivities some of the scalars were solved on a finer mesh than the flow-field (cf. §2.3). Parallelisation was achieved by dividing the computational domain into blocks of equal size. Each block was assigned to its own processing core in order to obtain a near-optimal load-balancing. The standard message passing interface (MPI) protocol was employed for communication between blocks. See Kubrak et al. (2013) and Herlina \& Wissink (2014) for a more detailed description of the numerical method. 


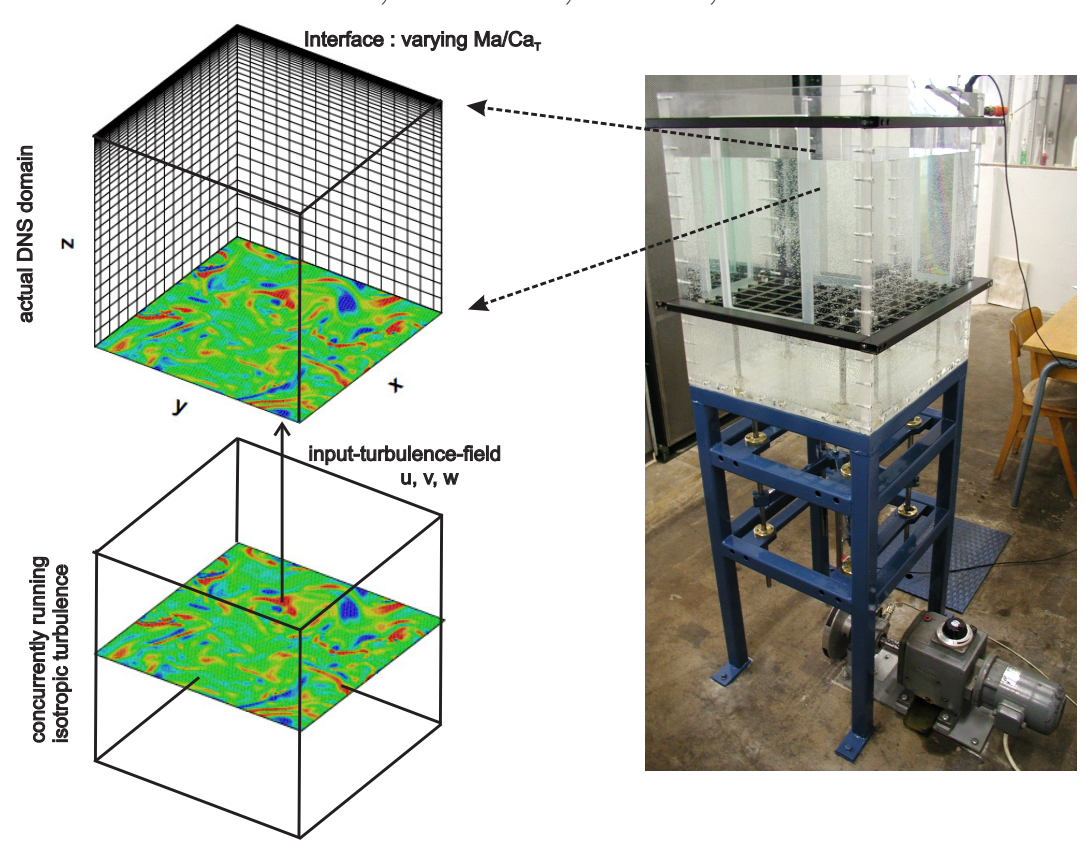

FiguRE 1. Schematic of the computational configuration (left pane). The configuration was selected in accordance with the grid-stirred turbulence driven gas transfer experiments (right pane) of Herlina \& Jirka (2008), where only a small part adjacent to the surface is modelled.

\subsection{Overview of simulations}

As in our previous simulations (Herlina \& Wissink 2014, 2016) the choice of the computational domain was based on the experiments performed by Herlina \& Jirka (2008) where turbulence was induced by an oscillating grid near the bottom of a water tank (cf. figure 1). In the present direct numerical simulations (DNS) a computational domain of size $L_{x} \times L_{y} \times L_{z}=5 L \times 5 L \times 3 L$ is employed, where the reference length scale $L$ is chosen arbitrarily to be $1 \mathrm{~cm}$. In all simulations, a Reynolds number of $R e=U L / \nu=600$ is selected, where - for a kinematic viscosity of $\nu=10^{-2} \mathrm{~cm}^{2} / \mathrm{s}$ - the reference velocity is $U=6 \mathrm{~cm} / \mathrm{s}$. Note that not Re but a turbulent Reynolds number $R_{T}$ - which is determined from the results - will be used to present and analyse the data below. Also, in the remainder of this paper - unless stated otherwise - all velocity, time, and length scales have been non-dimensionalised using $U$ and $L$. The flow is resolved using a $128 \times 128 \times 212$ base-mesh that is stretched in the $z$-direction to concentrate grid points near the interface (i.e. near $z=L_{z}$ ). The mass transfer calculation is performed for $S c=2,4,8,16,32$. The first two scalars are resolved using the base-mesh, while the latter three scalars are solved on a finer mesh with refinement factor 2. Exactly the same mesh was employed in our previous simulation GS200 in Herlina \& Wissink (2014), where an extensive grid refinement study was carried out to show the adequacy of the chosen resolution for both flow and scalar fields. Note that in the following analysis $\zeta=L_{z}-z$ will be additionally used to denote the distance from the free surface. To account for the much larger size of the experimental water tank, periodic boundary conditions are employed in the horizontal directions.

The input-turbulence at $z=0$ of the computational domain originated from a concurrently running large-eddy simulation (LES) of fully developed isotropic turbulence. A $64^{3}$ mesh is used to discretize the $\left(L_{x}\right)^{3}$ periodic box. Note that in Herlina \& Wissink 


$\begin{array}{lccccccc}\text { Run } & \text { S0 } & \text { S1 } & \text { S2 } & \text { S3 } & \text { S4 } & \text { S5 } & \text { SN } \\ M a / C a & 0 & 0.12 & 0.6 & 1.2 & 6 & 30 & \text { no-slip } \\ u_{\infty} & 0.1130 & 0.1119 & 0.1169 & 0.1085 & 0.1104 & 0.1114 & 0.1073 \\ L_{\infty} & 1.0333 & 0.9579 & 0.9935 & 0.9835 & 0.9273 & 1.0209 & 0.8984 \\ R_{T} & 141 & 128 & 139 & 131 & 125 & 138 & 117 \\ M a / C a_{T} & 0 & 1 & 5 & 11 & 54 & 269 & \text { no-slip }\end{array}$

TABLE 1. Overview of the simulations.

(2014), for a similar simulation, it was shown that the missing small scales in the energy spectrum of the LES quickly re-establish and a complete spectrum was obtained within a distance of one- $L$ from the bottom of the DNS which is well below the surface influenced layer. The turbulent flow-field introduced into the DNS domain is the same in all simulations. The turbulence level is set to $\sqrt{2\langle k\rangle / 3}=0.4$, where $k=\overline{u_{i}^{\prime} u_{i}^{\prime}} / 2$ is the turbulent kinetic energy, the prime denotes the fluctuating velocity, while $\langle\cdot\rangle$ and $\ulcorner$ correspond to averaging in the homogeneous directions and time, respectively. Note that in our DNS $u_{i}^{\prime}=u_{i}$.

In total seven simulations with varying $\mathrm{Ma} / \mathrm{Ca}$ numbers ranging from 0 (free-slip) to 30 as well as a no-slip case are performed (cf. table 1). Note that although in a hydrodynamics sense the contaminated surface will not converge to a no-slip surface, the scaling of mass transfer with $S c$ for the no-slip case agrees well with the scaling found for severely contaminated surface conditions where $\mathrm{Ma} / \mathrm{Ca}$ approaches $\infty$ (see $\S 4.2$ ). The boundary condition at the top depends on $\mathrm{Ma} / \mathrm{Ca}$ and affects the near surface turbulence, resulting in variations in the turbulent fluctuations

$$
u_{r m s}(\zeta)=\sqrt{\overline{\left\langle u^{\prime} u^{\prime}\right\rangle}}
$$

and the longitudinal integral length scale

$$
L_{11}(\zeta)=\int_{0}^{L_{x} / 2} R_{11}(r, \zeta) d r
$$

where the longitudinal two-point correlation $R_{11}$ of the horizontal velocity is defined by

$$
R_{11}(r, \zeta)=\frac{\int_{x=0}^{L_{x} / 2} \int_{y=0}^{L_{y}} u^{\prime}(x, y, \zeta) u^{\prime}(x+r, y, \zeta) d y d x}{\int_{x=0}^{L_{x} / 2} \int_{y=0}^{L_{y}} u^{\prime 2}(x, y, \zeta) d y d x},
$$

where $L_{x} \times L_{y}$ is the size of the horizontal plane. To allow a direct comparison between the different simulations, the characteristic turbulent velocity and length scales are all evaluated at the same location $\zeta=L$, which approximately corresponds to a distance equal to $L_{\infty}$ from the surface (cf. table 1 ), so that

$$
u_{\infty}=\left.u_{r m s}\right|_{\zeta=L}
$$

and

$$
L_{\infty}=\left.\overline{L_{11}}\right|_{\zeta=L} .
$$

The appropriateness of the location where $u_{\infty}$ and $L_{\infty}$ are evaluated is further explained 
in $§ 4.1$. Following the convention typically used for grid-stirred turbulence (e.g. Brumley \& Jirka 1987; Hopfinger \& Toly 1976), we use $\Lambda=2 L_{\infty}$ as the characteristic turbulent length scale. Using these scales, the turbulent Reynolds number reads

$$
R_{T}=\frac{u_{\infty} 2 L_{\infty}}{\nu}
$$

and the turbulent capillary number is given by

$$
C a_{T}=\frac{\mu u_{\infty}}{\sigma} .
$$

Note that the latter does not depend on the integral length scale. In the following analysis, the level of surface contamination will be characterised by the parameter $M a / C a_{T}$ and time averaging is performed from $t=150$ to $t=300$, corresponding to approximately seven eddy turnover times $\left(2 L_{\infty} / u_{\infty}\right)$.

Khakpour et al. (2011) showed that the actual Schmidt number of the surfactant is not important for an accurate calculation of the surfactant concentration. Hence, it is decided to perform all simulations using a surfactant Schmidt number of $S c_{s}=2$.

\section{Proposed estimation of mean $K_{L}$ based on clean surface fraction $\bar{\alpha}$}

As mentioned above, it is likely that the exponent $q$ in (1.6) will depend on the level of surface contamination. Based on this idea, we propose an estimation for both $q$ and $c$ by using the average clean surface fraction $\bar{\alpha}$ (suitably defined by means of a threshold, cf. $\S 4.2 .4$ ), which is likely to be a relatively "easy observable" parameter. Our proposed model for $c S c^{-q}$ in (1.6) reads

$$
c S c^{-q}=\bar{\alpha} c_{f} S c^{-q_{f}}+(1-\bar{\alpha}) c_{n} S c^{-q_{n}}
$$

with $q_{f} \leqslant q \leqslant q_{n}$, where $q_{f}, c_{f}$ and $q_{n}, c_{n}$ correspond to the exponents of $S c$ and the constants of proportionality for the free-slip and no-slip cases, respectively. To obtain expressions for $c$ and $q$ based on $\bar{\alpha}$ we substitute the following Taylor expansions

$$
\begin{aligned}
& S c^{-q_{f}}=S c^{-\left(q-h_{1}\right)} \approx S c^{-q}+h_{1}(\ln S c) S c^{-q}+O\left(h_{1}^{2}\right) \\
& S c^{-q_{n}}=S c^{-\left(q+h_{2}\right)} \approx S c^{-q}-h_{2}(\ln S c) S c^{-q}+O\left(h_{2}^{2}\right)
\end{aligned}
$$

into (3.1) and after ignoring the second and higher order terms, we obtain :

$$
c S c^{-q}=\left[\bar{\alpha} c_{f}\left(1+h_{1} \ln S c\right)+(1-\bar{\alpha}) c_{n}\left(1-h_{2} \ln S c\right)\right] S c^{-q}
$$

Assuming $c$ is independent of $S c$, it is necessary to eliminate $\ln S c$ from this expression, which is achieved when

$$
\bar{\alpha} c_{f} h_{1}-(1-\bar{\alpha}) c_{n} h_{2}=0 .
$$

If we replace $h_{1}$ by $\Delta_{n f}-h_{2}$, where $\Delta_{n f}=q_{n}-q_{f}$ we obtain

$$
\begin{aligned}
h_{2} & =\frac{\Delta_{n f} \bar{\alpha} c_{f}}{(1-\bar{\alpha}) c_{n}+\bar{\alpha} c_{f}} \\
q & =q_{n}-h_{2} \\
c & =\bar{\alpha} c_{f}+(1-\bar{\alpha}) c_{n}
\end{aligned}
$$

In accordance to the literature, the exponents $q_{f}$ and $q_{n}$ are fixed to $1 / 2$ and $2 / 3$, respectively. The parameters $c_{f}$ and $c_{n}$ will be determined from results of the free-slip and no-slip cases. Subsequently, $K_{L} /\left(u_{\infty} R_{T}^{-r}\right)$ can be predicted as a function of $\bar{\alpha}$ alone using (3.5), (3.7) and (1.6). The quality of the proposed prediction will be investigated in $\S 4.4$. 


\section{Results}

\subsection{Turbulent flow statistics}

Before discussing the effect of surface contamination levels on interfacial mass transfer, we will briefly discuss its influence on turbulent flow statistics. As mentioned in $\S 2.3$, in all seven simulations the same turbulent flow-field is introduced at $z=0$, whilst at the surface $\left(z=L_{z}\right)$ different levels of contamination are imposed by varying $M a / C a_{T}$. Figures 2a, b illustrate the decay of the turbulence as it diffuses upwards. For all simulations, the levels of horizontal (surface-parallel) and vertical (surface-normal) fluctuations in the lower part of the computational domain (up to $z \approx 1.5 L$ ) were found to be almost identical, while up to $z=2 L$ the flow was still found to be largely isotropic. Closer to the surface (i.e. in the surface influenced layer) differences become noticeable. Responsible for this are the different boundary conditions at the surface, where the vertical velocity is always zero, while the horizontal velocities depend on $M a / C a_{T}$. As a consequence, the variation in the level of vertical fluctuations is much smaller than in the horizontal fluctuations. In agreement with previous numerical results (e.g. Perot \& Moin 1995; Walker et al. 1996; Calmet \& Magnaudet 2003), for the free-slip case (S0) $w_{r m s}$ is found to suddenly decrease towards the surface while $u_{r m s}$ simultaneously increases (a more detailed discussion can be found in Herlina and Wissink 2014).

Figure 2c shows a more detailed view of the $u_{r m s}$ profiles near the surface. With the exception of the no-slip simulation (SN), the horizontal fluctuation levels were observed to coincide reasonably well up to $z \approx 2.8 L$ (or $\zeta \approx 0.2 L$ ). The different result obtained for $\mathrm{SN}$ is due to the application of a no-slip boundary condition at the surface, while in the other simulations the surface velocity field is merely forced to become more and more divergence free with increasing $M a / C a_{T}$. From $z \approx 2.8 L$ to the surface the horizontal fluctuations in S0 and S1 were found to increase, while in all other simulations $u_{r m s}$ decreases. In general, it is observed that $u_{r m s}$ in S0 to S5 tends to slightly decrease with increasing $M a / C a_{T}$, whereby the results from $\mathrm{S} 0$ and $\mathrm{S} 1$ as well as $\mathrm{S} 4$ and $\mathrm{S} 5$ almost coincide. This decrease in $u_{r m s}$ illustrates that rising levels of surface contamination tend to increase near-surface turbulence damping. The increase in $u_{r m s}$ observed in S0 and S1 is caused by the redistribution of turbulent kinetic energy: close to the surface the vertical velocity fluctuations decline and as a result the horizontal fluctuations are enlarged (see also Perot \& Moin 1995). The aforementioned rising levels of surface contamination result in increased instantaneous shear and, hence, in increased damping of $u_{r m s}$ as can be seen in figure 2c for S2 - S5.

Figure $2 \mathrm{~d}$ shows that the $w_{r m s}$ profiles exhibit a similar decreasing trend with $\mathrm{Ma} / \mathrm{Ca}_{T}$ as observed for $u_{r m s}$ above. This decrease in $w_{r m s}$ is expected to affect the interfacial mass transfer, which is further investigated in $\S 4.2 .2$ below. Note that the $w_{r m s}$ profiles from both $\mathrm{S} 0$ and $\mathrm{S} 1$ as well as $\mathrm{S} 5$ and $\mathrm{SN}$ almost coincide. It can be easily seen from Taylor series expansions that close to the surface $w_{r m s}$ is proportional to $\zeta^{2}$ in the noslip case, while in the free-slip case $w_{r m s} \propto \zeta$. Both trends are consistently reproduced by our simulations $\mathrm{SN}$ and $\mathrm{S} 0$, respectively. The near surface $w_{r m s}$ in simulations S1 to S4 $\left(0<M a / C a_{T} \leqslant 54\right)$ is found to behave similarly to the free-slip simulation with $w_{r m s} \propto \zeta$. Only in S5 where $M a / C a_{T}=269$ an intermediate behaviour between free-slip and no-slip is found with $w_{r m s} \propto \zeta^{1.36}$.

With the exception of the no-slip simulation $\mathrm{SN}$, all cases have a nonzero 2D velocity field at the surface. While in the free-slip simulation the velocity field quickly becomes $3 \mathrm{D}$ with increasing depth $($ as $w \propto \zeta)$, in cases with large $M a / C a_{T}$ this takes much longer as $w \propto \zeta^{2}$. The latter is a result of the Marangoni effect - caused by horizontal gradients in the surfactant concentration - inducing a force counteracting the aforementioned sur- 


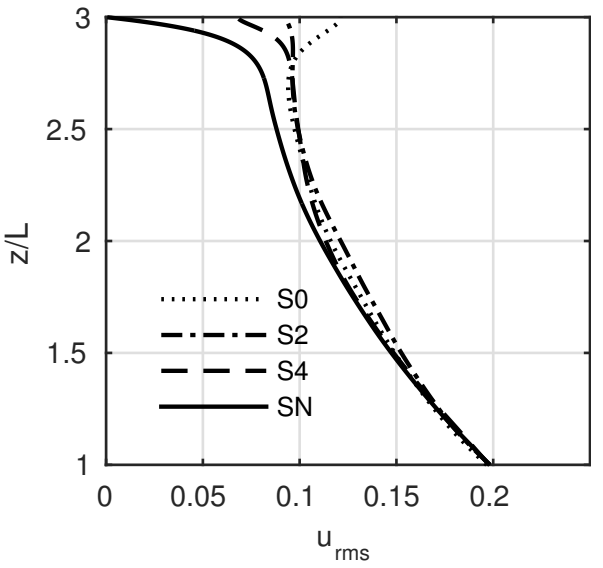

(a)

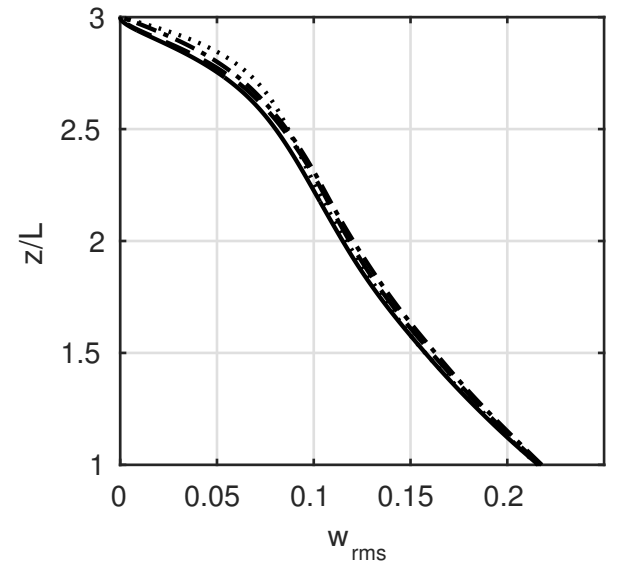

(b)

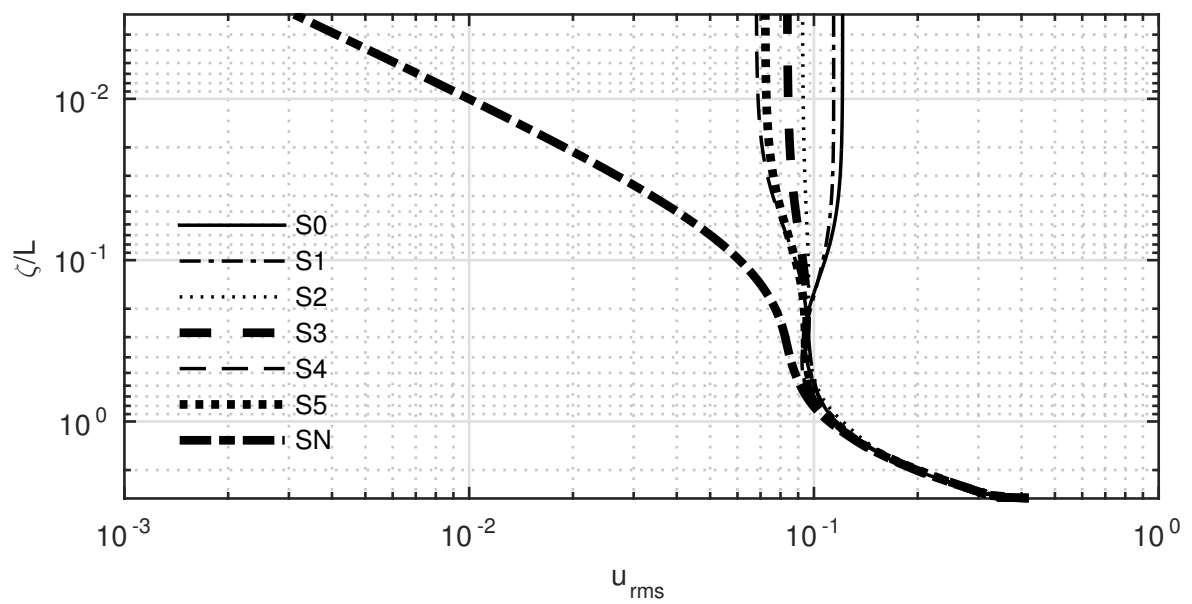

(c)

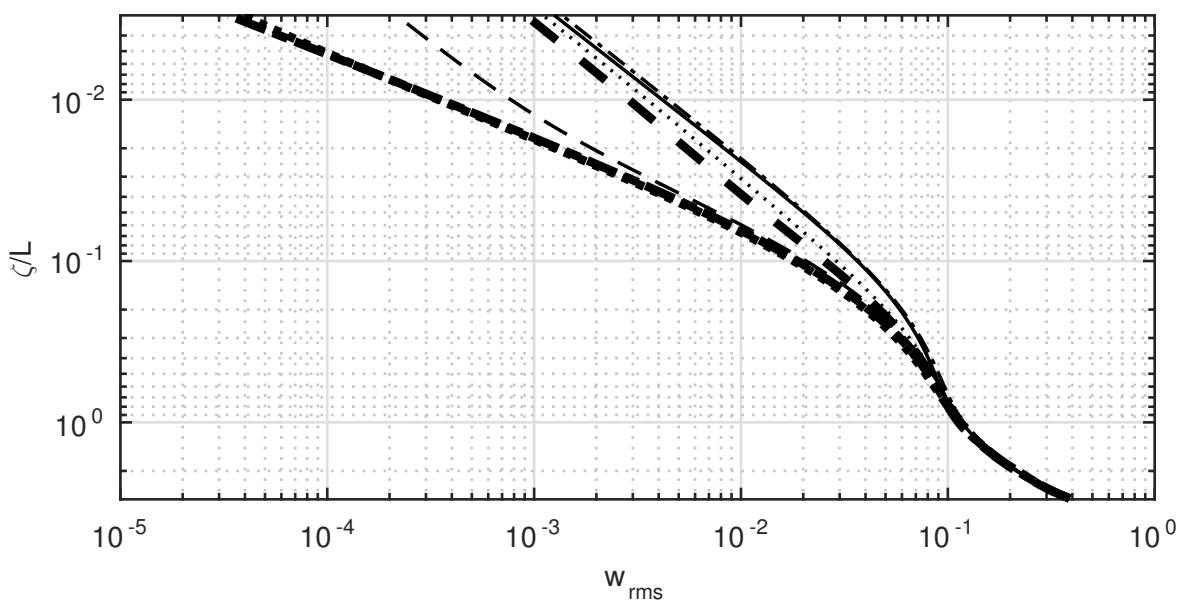

(d)

Figure 2. Effect of $M a / C a_{T}$ on the near-surface turbulent flow statistics : (a) $u_{r m s}$, (b) $w_{r m s}$. (c-d) the same as (a-b) plotted in logarithmic scale and using the inverse coordinate $\zeta=L_{z}-z$. Shown are time-averaged $(t=150$ to 300$)$ results. 


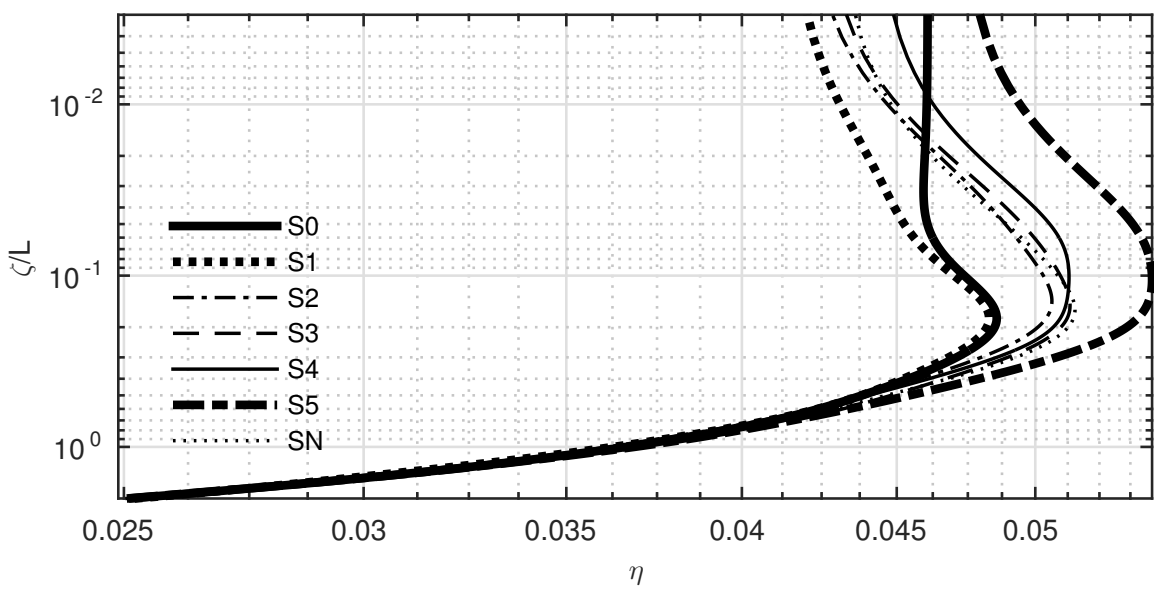

(a)

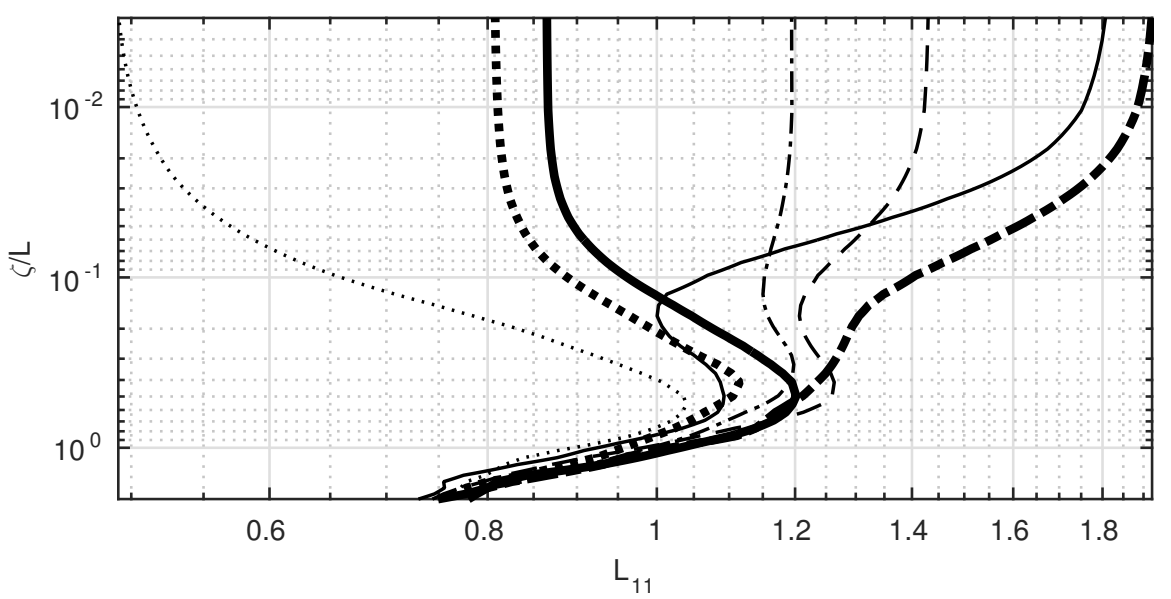

(b)

FiguRE 3 . Vertical variation of (a) turbulent integral length scale $L_{11}$ (time-averaged from $t=150$ to 300 ), and (b) Kolmogorov length scale $\eta$ at $t=300$.

factant gradients thereby effectively forcing the $2 \mathrm{D}$ flow at the surface to become almost divergence free $(\partial u / \partial x+\partial v / \partial y \approx 0)$.

For all simulations, the turbulent integral length scale $L_{11}$ shown in figure $3 \mathrm{a}$ is first found to increase with distance from the turbulent source until a local maximum is reached at $\zeta \approx 0.5 L$. Above this location $L_{11}$ reduces to zero at the surface in the noslip case, while in the free-slip simulation and in the low $M a / C a_{T}$ case (S1) it reduces to approximately 0.87 and 0.8 , respectively. In $\mathrm{S} 2$ to $\mathrm{S} 5$ (moderate to large $\mathrm{Ma} / \mathrm{Ca} \mathrm{a}_{T}$ ) the integral length scale is found to increase again when approaching the surface. An explanation of this behaviour is given in the discussion of figure 4 below. It is attributed to the presence of instantaneous shear which becomes stronger with increasing $M a / C a_{T}$ such that for S2 to S5 an increased horizontal integral length scale is obtained.

Figure $3 \mathrm{~b}$ shows the variation of the Kolmogorov scale $\eta=\left(\nu^{3} / \epsilon\right)^{1 / 4}$ with distance from the surface for $t=300$. Note that $\epsilon$ is calculated by

$$
\epsilon=2 \nu s_{i j} s_{i j}
$$

where $s_{i j}=\frac{1}{2}\left(\partial u_{i}^{\prime} / \partial x_{j}+\partial u_{j}^{\prime} / \partial x_{i}\right)$. Apart from S0, it can be seen that $\eta$ increases 
monotonically from the lower bulk upwards until it reaches a maximum at $\zeta$ between $0.1 L$ and $0.2 L$. For $\zeta$ approaching zero two trends can be observed: (i) in $\mathrm{S} 0 \eta$ becomes constant, and (ii) in all other cases $\eta$ decreases, indicating vortex stretching which is most likely caused by instantaneous shear forces. In SN this shear is caused by the noslip boundary condition, while in S1 to S5 it is induced by the Marangoni forces.

Using representative snapshots the effect of shearing on the interfacial integral length scale $L_{11}$ is illustrated by visualizing the vortical structures close to the surface (cf. figure 4) using the $\lambda_{2}$ criterion of Jeong \& Hussain (1995). Compared to S4 and S5, in S0 significantly fewer vortical structures managed to reach the surface whereby the axes of these structures were found to be orthogonal to the surface. In S4 and S5 both the diameter and the orientation of a significant proportion of the vortex tubes that reach the surface differ from the ones observed in S0. In S0 the vortex tubes have a constant diameter and are orientated perpendicular to the surface. The former is in agreement with the constant $\eta$ between $\zeta=0.1$ to 0 as observed in figure 3b. Contrary, in S4 and S5 many vortex tubes are non-orthogonal to the surface such that their horizontal crosssectional area widens towards the surface, which may contribute to a larger $L_{11}$ at the surface. This non-orthogonality can be explained by the instantaneous shear induced by the Marangoni forces at the surface. Note that the apparent widening of the horizontal cross-sectional area of vortex tubes towards the interface in S4 and S5 does not imply that the actual diameter of the vortex tubes increases. Instead the diameter of the tubes is known to scale with the Kolmogorov length scale which was observed to decrease towards the surface as shown in figure 3b. In contrast to the no-slip simulation SN - where it is impossible for vortices to reach the surface as the velocity field is zero - the vortex structures in S4 and S5 do reach the surface indicating that the case $M a / C a_{T} \rightarrow \infty$ is hydrodynamically different from the no-slip case.

Figure 5 shows the turbulent Reynolds number profiles of S0, S2, S4, SN. In agreement with earlier studies of grid-stirred generated turbulence diffusing upwards, eventually the horizontal turbulent fluctuations $u_{r m s}$ are observed to scale with $z^{-1}$, while the integral length scale $L_{11}$ increases with $z$ (e.g. Brumley \& Jirka 1987). Consequently, their product - and hence $R_{T}$ - becomes constant, which in our simulations is achieved between $z=1.5 \mathrm{~L}$ and $2.25 \mathrm{~L}$, illustrating the appropriateness of our choice to evaluate $L_{\infty}$ and $u_{\infty}$ at $z=2 L$. Note that this location corresponds to the edge of the surface influenced layer (cf. figure 2).

\subsection{Influence of $M a / C a_{T}$}

\subsubsection{Qualitative observations}

In figure 6 the instantaneous concentration isosurfaces for $c_{S c=4}=0.5$ from simulations $\mathrm{S} 1, \mathrm{~S} 2$ and S4 are shown. The isosurfaces are coloured by the corresponding surfactant concentration at the surface $\gamma(x, y)$ which is normalised using the maximum instantaneous concentration $\gamma_{\max }$ (see also (4.4) below). The plots illustrate the dynamic variation in concentration boundary layer thickness induced by the turbulence from below as discussed previously for clean surfaces by e.g. Magnaudet \& Calmet (2006); Nagaosa \& Handler (2003) and for clean and severely contaminated surfaces by e.g. Khakpour et al. (2011). Generally, at the locations where the boundary layer is thin, surfactants are pushed to the side by strong upwelling motions (splats) and subsequently accumulate in the downwelling (antisplats) regions identified by boundary layer thickening. In agreement with the enhanced turbulence damping for increased levels of contamination observed in figure 2, the downwelling is observed to be strongest in S1 as can be seen by the deep penetration of the antisplats into the bulk. Furthermore, in this low $M a / C a_{T}$ simulation strong upwelling motions result in a large virtually surfactant-free surface frac- 


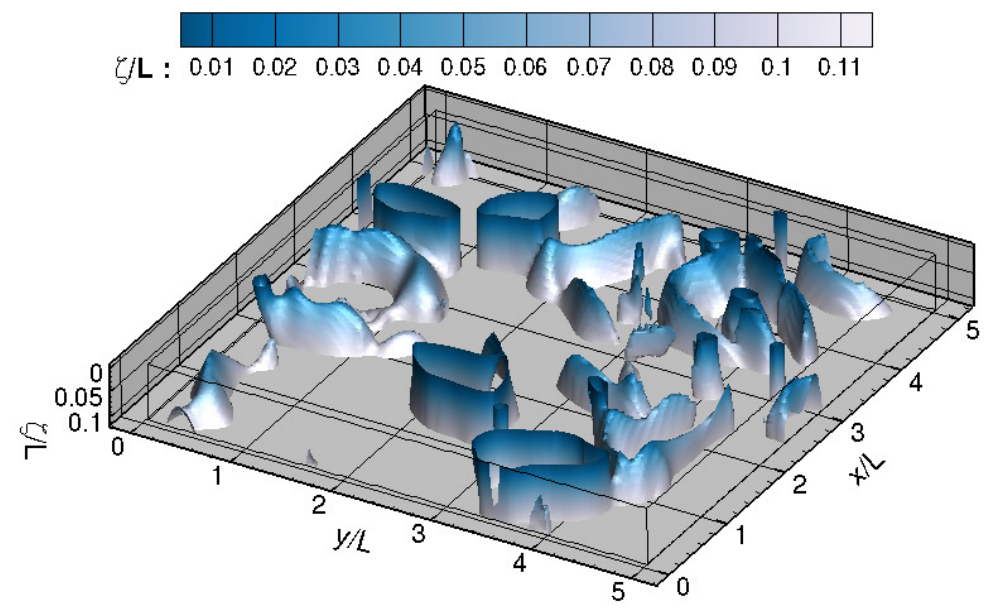

(a)

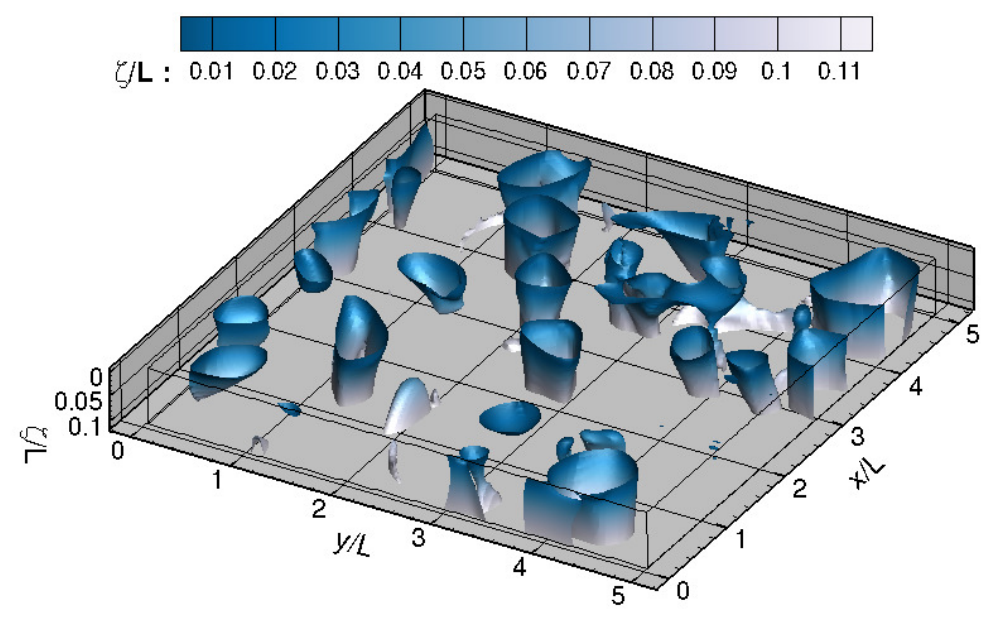

(b)

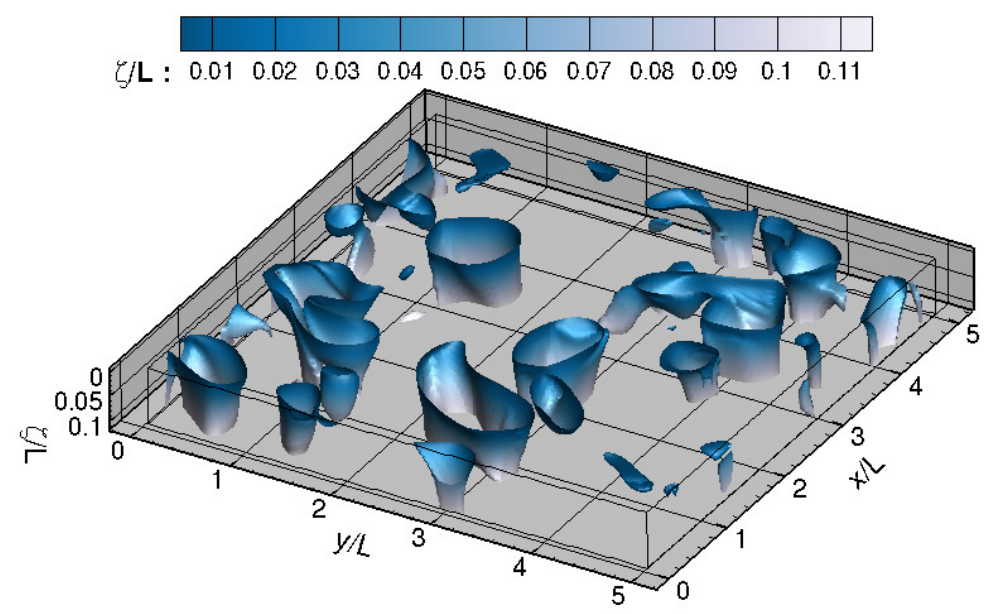

(c)

FiguRE 4. Vortical structures identified using the isosurface of $\lambda_{2}=-0.001$ from simulations (a) S0, (b) S4, (c) S5. The isosurfaces are coloured by the distance $\zeta$ from the surface. 


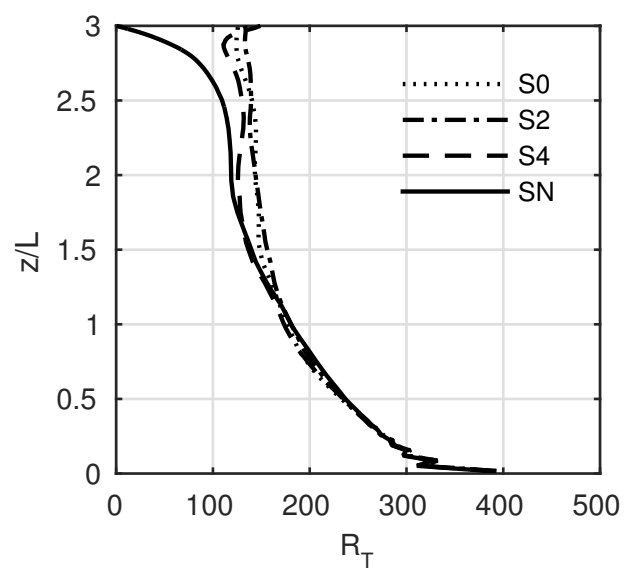

Figure 5. Effect of $M a / C a_{T}$ on $R_{T}$. Shown are time-averaged ( $t=150$ to 300 ) results.

tion (figure 6a). For increasing $M a / C a_{T}$ this surface fraction becomes rapidly smaller and is found to completely disappear in S4. The connection between $M a / C a_{T}$ and the surfactant-free area will be further investigated below (cf. $\S$ 4.2.4).

Figure 7 contrasts the correlation between the instantaneous concentration (colour contours) and the surface divergence $\beta$ (isolines), defined by

$$
\beta=\left.\left(\frac{\partial u}{\partial x}+\frac{\partial v}{\partial y}\right)\right|_{z=L_{z}},
$$

in the grid-plane adjacent to the surface in simulations S1 and S5. In S1 areas of low concentration are observed to coincide with strong positive surface divergence indicating upwelling, while in areas of high concentration the surface divergence is found to be negative. In S5, on the other hand, the above correlation becomes less clear and areas with strong positive surface divergence are found not to always coincide with low concentration levels and vice versa. In general, when upwelling first occurs the correlation between low concentration and strong positive surface divergence is found to be very good. At large $M a / C a_{T}$ (e.g. S5), surface divergence tends to induce strong Marangoni forces that in turn act to reduce surface divergence. As a result, in time the correlation between low concentration regions and strong positive surface divergence is largely lost and instantaneous shear is generated near the surface, as also observed in figure 4 (see also Khakpour et al. 2011; Handler et al. 2003). Note that in this respect the high $M a / C a_{T}$ simulations S4 and S5 behave like the no-slip case SN.

\subsubsection{Vertical mass flux}

Figure 8 shows the effect of $M a / C a_{T}$ on the mean vertical concentration, the rms of the concentration fluctuations, and the diffusive and turbulent mass flux profiles from simulations S0, S1, S2, S3 and SN. Note that the profiles from simulations S4 and S5 (not shown here) were found to be almost identical with the SN profiles. Figure 8a depicts the normalised mean concentration profiles

$$
\frac{\overline{\langle c\rangle}-\overline{\left\langle c_{b}\right\rangle}}{c_{s}-\overline{\left\langle c_{b}\right\rangle}}
$$

where $c_{b}$ is evaluated in the bulk at $z=z_{b}$, chosen such that

$$
c_{r m s}\left(z_{b}\right)=0.5 \max \left(c_{r m s}\right) \text {. }
$$




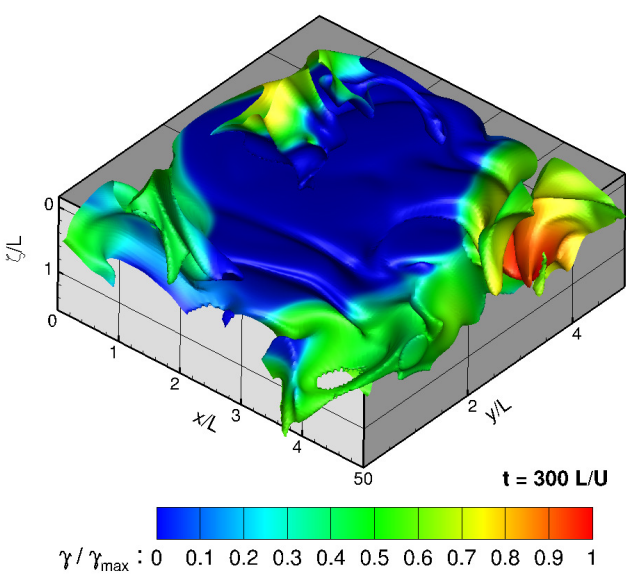

(a)

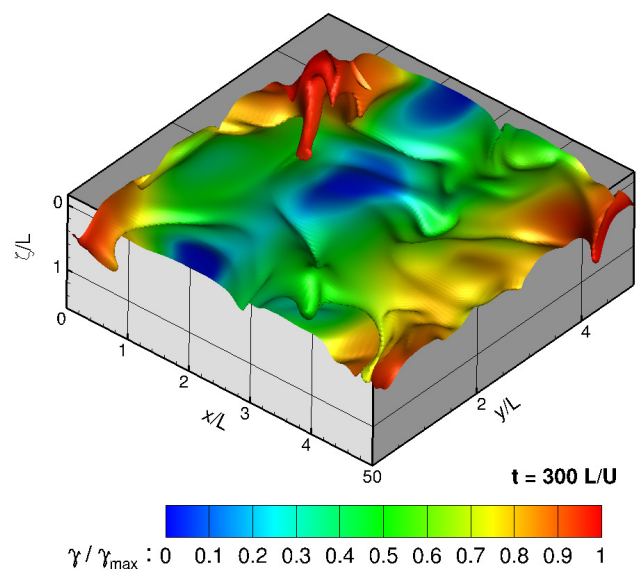

(b)

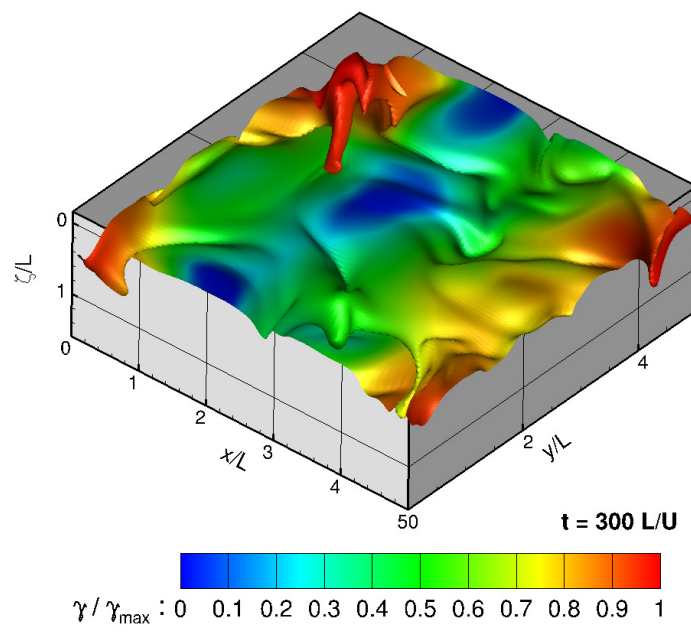

(c)

FigURE 6. Instantaneous isosurfaces of concentration at $c_{S c=4}=0.5$ from (a) S1, (b) S2 and (c) S4 at $t=300 L / U$. The colours represent the normalised surfactant concentration at the corresponding interfacial $(x, y)$ coordinates.

It can be seen that an increase in the contamination level leads to a thickening of the mean concentration boundary layer. The thickness $\delta$ of this layer can be identified by the depth where $c_{r m s}$ reaches its maximum. In agreement with the above observation, in figure $8 \mathrm{~b}$ the peak in $c_{r m s}$ is seen to move farther from the surface with increasing $\mathrm{Ma} / \mathrm{Ca}_{T}$. This thickness $\delta$ was found to gradually increase from $0.0239,0.0273,0.0345$, 0.0424, 0.0487 for S0, S1, S2, S3, and SN, respectively. Also, with the exception of S0, the peaks in $c_{r m s}$ are found to decrease with increasing surface contamination level. The relatively large peak observed in S1 compared to S0 is associated with the stronger up and downwellings caused by an increase in the surface divergence fluctuations $\left(\beta_{r m s}\right)$ in $\mathrm{S} 1$ which is explained in more detail in $\S 4.2 .3$, cf. discussion of figure $8 \mathrm{~b}$.

The total vertical mass flux is determined by the sum of the vertical diffusive $\overline{\langle-D \partial c / \partial z\rangle}$ 


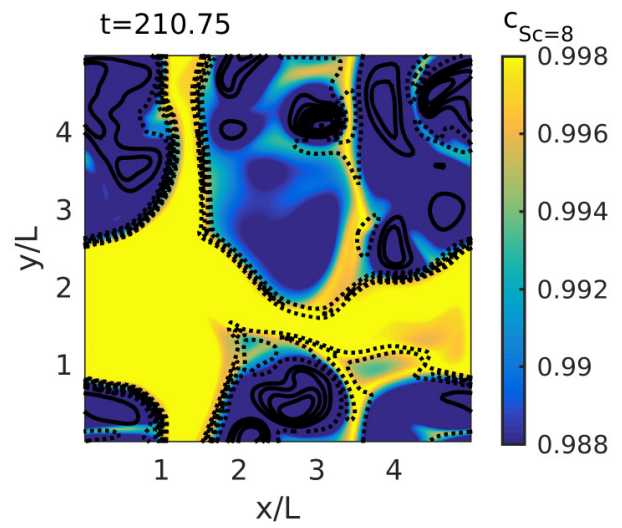

(a)

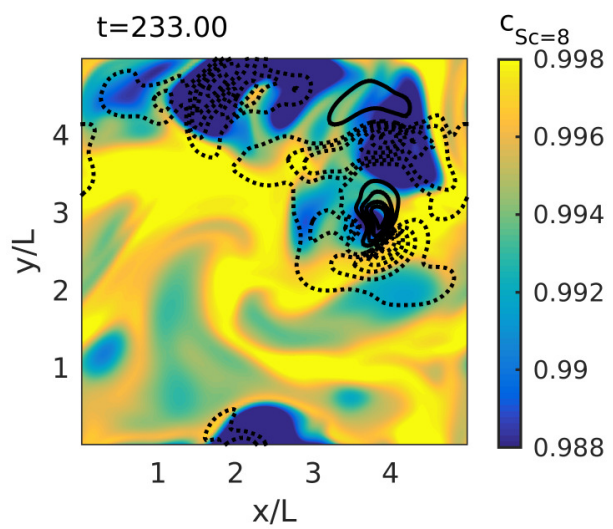

(b)

FiguRE 7. Effect of increasing $M a / C a_{T}$ on the correlation between the dissolved gas concentration (colour contours) and surface divergence (isolines). The solid and dotted lines correspond to positive and negative surface divergence, respectively. Snapshots are from (a) S1 and (b) S5.

and turbulent $\overline{\left\langle c^{\prime} w^{\prime}\right\rangle}$ mass fluxes. At the surface the mass flux is entirely due to diffusion. Above it was observed that the boundary layer thickness decreases with decreasing $M a / C a_{T}$, as a result the gradients $\overline{\partial c / \partial z}$ become steeper leading to an increase of the interfacial mass flux (figure 8c). Note that for the present no-slip simulation with $R_{T}=117$ this gradient remains constant up to $\zeta / \delta \approx 0.2$. As a consequence, diffusion fully dominates mass flux not only at the surface but also immediately beneath. This is in agreement with figure $8 \mathrm{~d}$ where the normal gradient of the scalar flux $\overline{\left\langle c^{\prime} w^{\prime}\right\rangle}$ vanishes at the surface. The latter immediately follows from the fact that at the surface (i) $w^{\prime}=0$ and (ii) the conservation of mass requirement for the no-slip simulation implies that $\partial w^{\prime} / \partial z=0$. For larger $R_{T}$ the distance from the surface for which the above applies reduces, as can be seen in figure 11c of Herlina \& Wissink (2016), where for $R_{T}=865$ the gradient is only approximately constant up to $\zeta / \delta \approx 0.08$.

Figures $8 \mathrm{c}, \mathrm{d}$ also show that with increasing distance from the surface the diffusive mass flux rapidly reduces, while simultaneously the turbulent mass flux increases so that the total vertical mass flux is kept constant. Already at a depth of three times the boundary layer thickness the turbulent mass flux almost completely dominates the total mass flux. In agreement with the reduction in the diffusive mass flux at the surface, figure $8 \mathrm{~d}$ shows that the turbulent mass flux in the bulk also reduces with increasing level of contamination.

Figure 9 illustrates the influence of the surface contamination level on the mean transfer velocity $K_{L}=\overline{k_{L}}$, where the instantaneous $k_{L}$ is defined by

$$
k_{L}(t)=\frac{\left\langle-\left.D \frac{\partial c}{\partial z}\right|_{z=L_{z}}\right\rangle}{c_{s}-\left\langle c_{b}\right\rangle},
$$

with $D=\frac{1}{R e S c}$. It can be seen that the average transfer velocity $K_{L}$ reduces drastically within a narrow range of $M a / C a_{T}=0$ to 11 . For $M a / C a_{T} \geqslant 54$ the time averaged transfer velocity is found to be similar to the no-slip case. Note that because the resulting $R_{T}$ in the simulations varies between 114 and 141 - which is well within the region where 


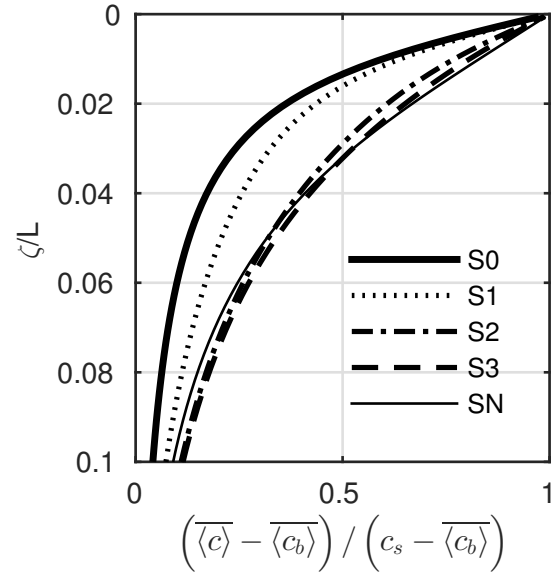

(a)

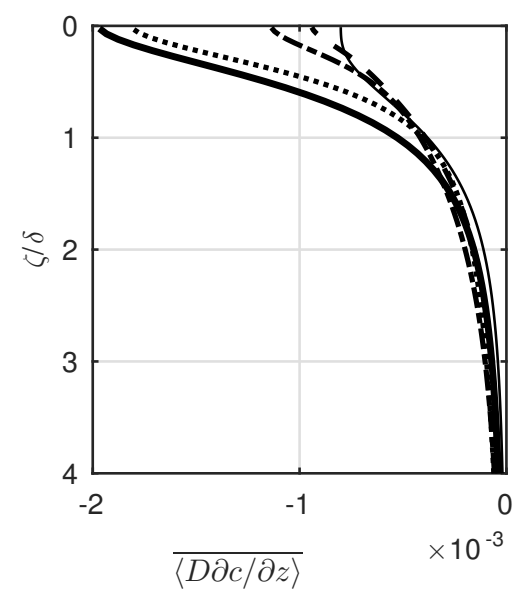

(c)

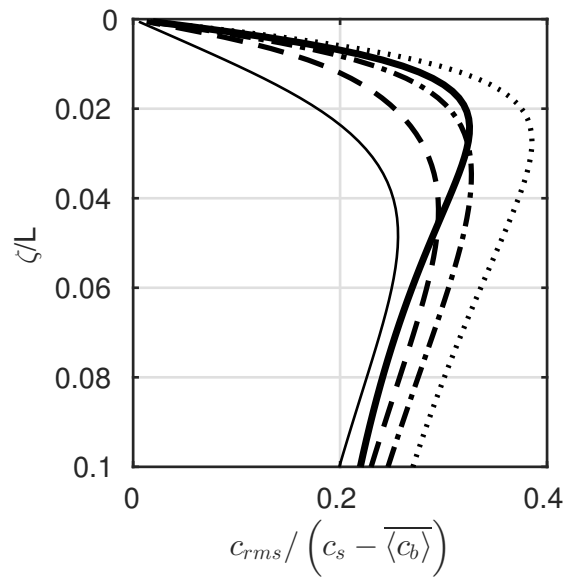

(b)

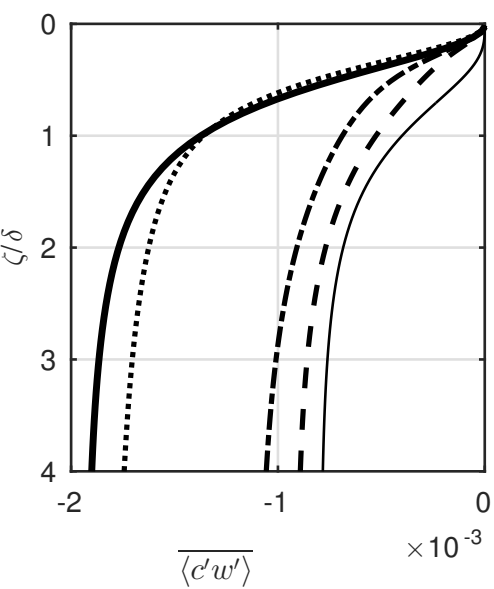

(d)

FIGURE 8. Effect of $M a / C a_{T}$ on the horizontally and time averaged profiles of (a) normalised concentration, (b) normalised concentration fluctuations, (c) vertical scalar diffusion, (d) vertical turbulent scalar flux, In (c) and (d) the depth $\zeta$ is normalised using the boundary layer thickness. Note that the molecular diffusion coefficient $D$ is defined by $D=\frac{1}{R e S c}$.

the large-eddy model is valid (Theofanous et al. 1976; Herlina \& Wissink 2016) - $K_{L}$ is normalised by $R_{T}^{-1 / 2}$ in order to allow for a direct comparison of the results, as discussed in the introduction.

In figure 10 the variation of $K_{L}$ with Schmidt number is shown. It can be seen that for each individual $\mathrm{Ma} / \mathrm{Ca}_{T}$ case there is a power law dependency $K_{L} \propto S c^{-q}$. When $\mathrm{Ma} / \mathrm{Ca}$ increases from 0 to 54 , the exponent $q$ is found to gradually increase from $1 / 2$ to $2 / 3$. Beyond $M a / C a_{T}=54, q$ is observed to remain constant at $2 / 3$. In Herlina \& Wissink (2014) and Herlina \& Wissink (2016) the above scaling of the transfer velocity with $S c^{-1 / 2}$ and $S c^{-2 / 3}$ for the free-slip $\left(M a / C a_{T}=0\right)$ and no-slip cases, respectively, was confirmed for $S c$ up to 500 . Therefore, it is expected that also for cases $M a / C a_{T}>0$ the obtained exponent $q$ in $K_{L} \propto S c^{-q}$ remains valid up to at least $S c=500$. 


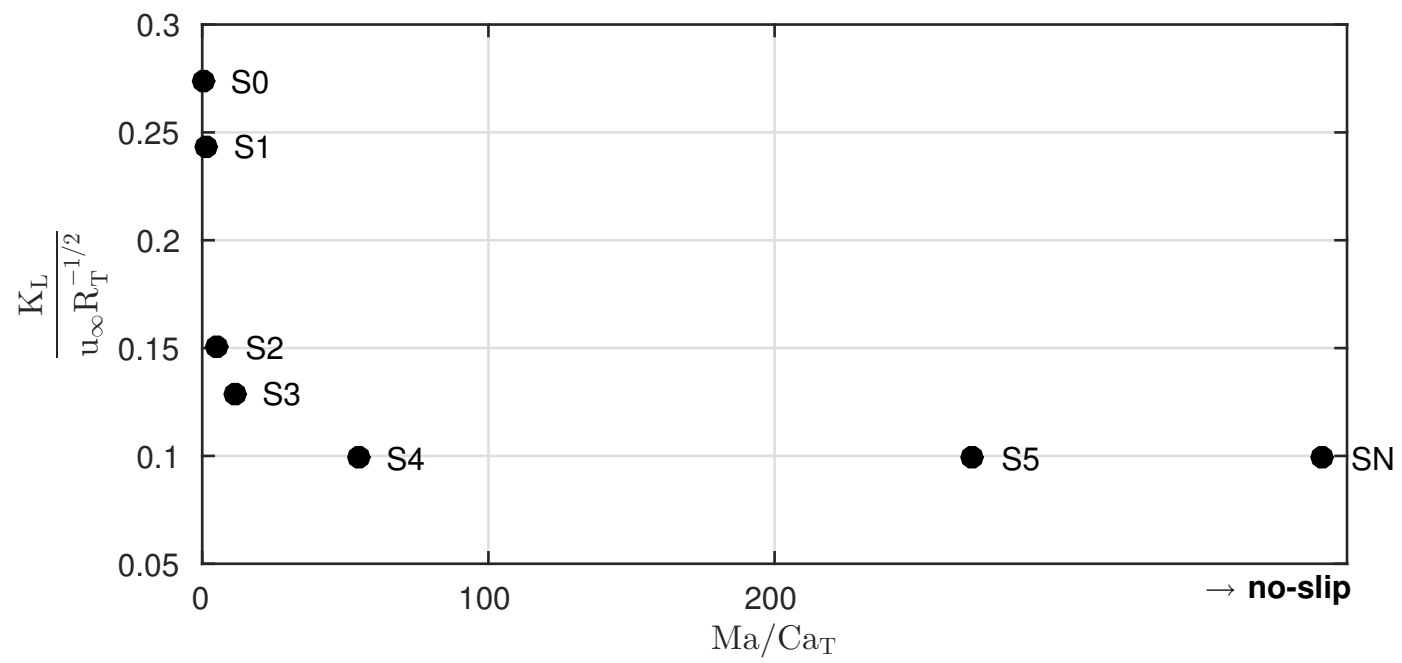

Figure 9. Variation of $K_{L}$ with $M a / C a_{T}$ for $S c=32$.

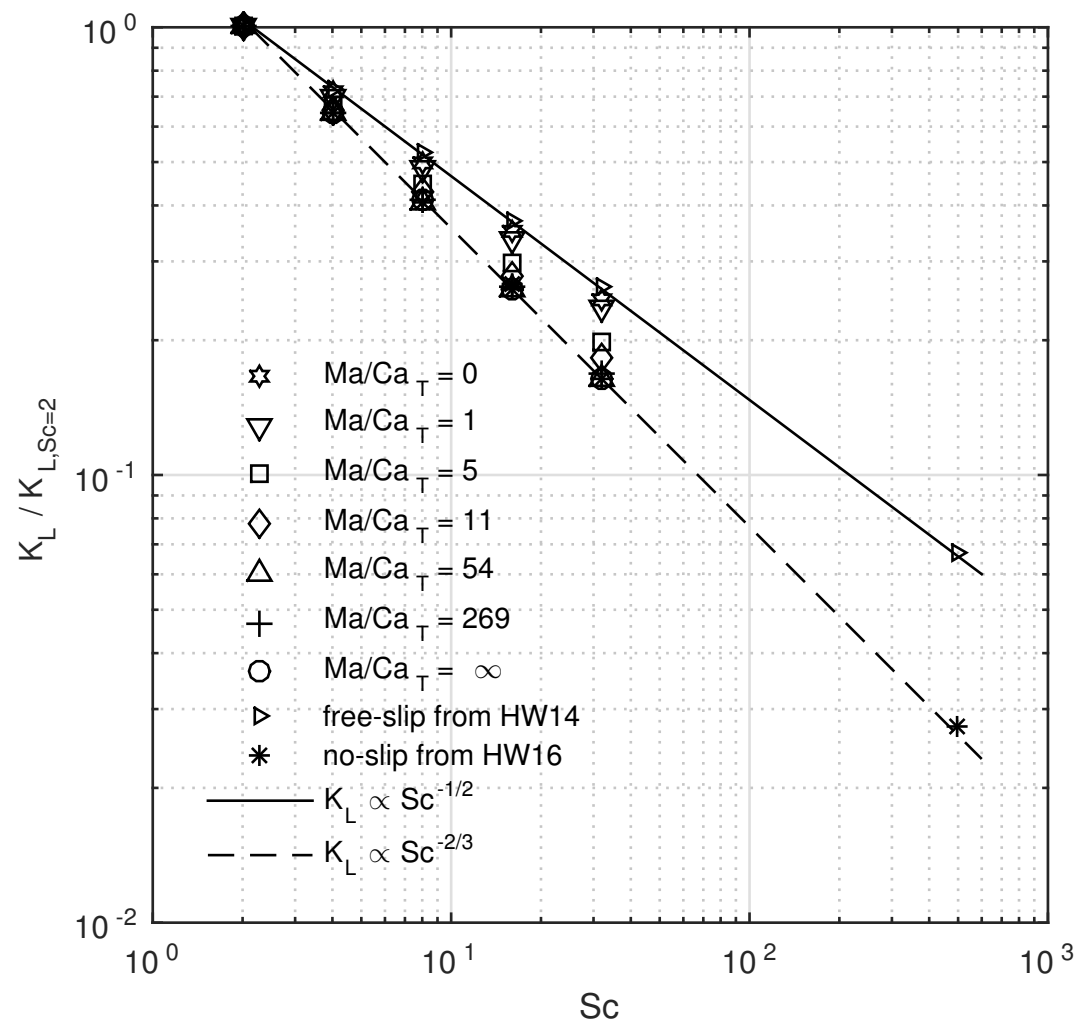

Figure 10. $K_{L}$ vs $S c$ for a range of $M a / C a_{T}$ (see table 1), HW14 and HW16 are taken from Herlina \& Wissink (2014) and Herlina \& Wissink (2016), respectively

\subsubsection{Surface divergence}

Figure 11a shows contours of the instantaneous surface divergence $\beta$ - defined in (4.1) - for the cases S0,S2,S4. It can be seen that the size of the area with virtually nonzero 

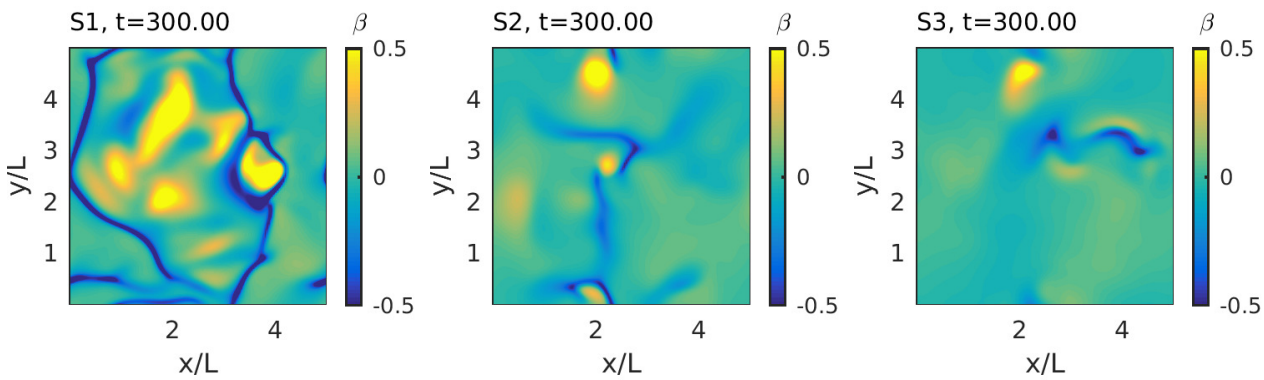

(a)

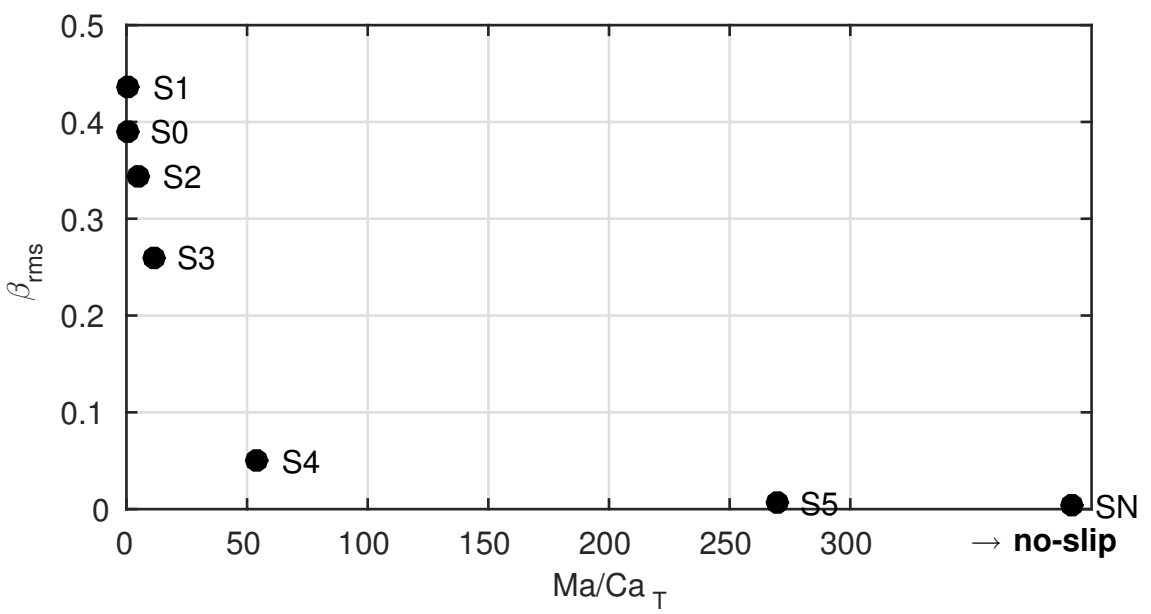

(b)

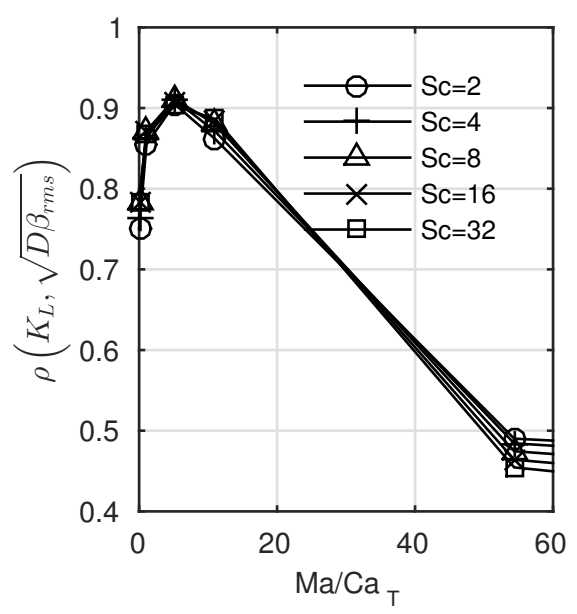

(c)

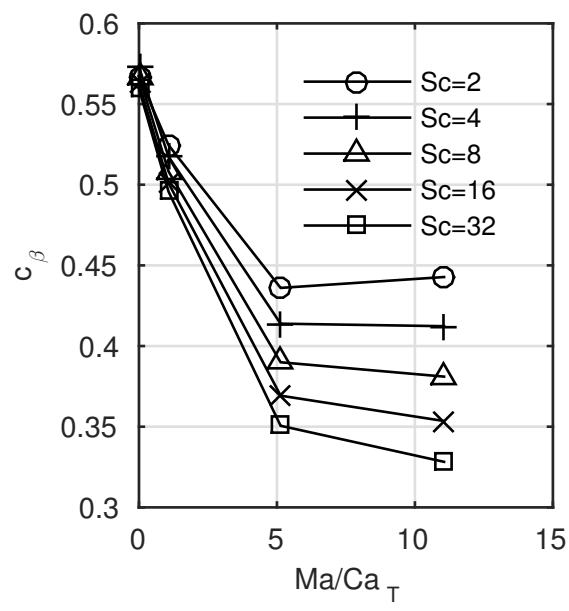

(d)

FiguRE 11. Effect of increasing $M a / C a_{T}$ on: a) snapshots of surface divergence, b) $\beta_{r m s}$, c) correlation coefficient of $K_{L}$ and $\sqrt{D \beta_{r m s}}$ and d) $c_{\beta}$. 
divergence decreases with increasing $M a / C a_{T}$ and, hence, it is to be expected that $\beta_{\text {rms }}$ also decreases. The variation of $\beta_{r m s}$ with $M a / C a_{T}$ is shown in figure $11 \mathrm{~b}$. As also observed previously by Shen et al. (2004), in general $\beta_{r m s}$ is indeed found to reduce sharply, approaching zero within a very small $M a / C a_{T}$ range. This abrupt reduction in $\beta_{r m s}$ towards zero implies that the surface divergence model would only work for a very small range of $\mathrm{Ma} / \mathrm{Ca}_{T}$, as the model will break down when $\beta_{r m s}$ approaches zero, as illustrated by the steep decline in the correlation coefficient between $K_{L}$ and $\sqrt{D \beta_{\text {rms }}}$ for large $\mathrm{Ma} / \mathrm{Ca}_{T}$ (cf. figure 11c).

Also, it can be seen in figure $11 \mathrm{~b}$ that $\beta_{r m s}$ in S1 is larger than in S0. This rather unexpected result is caused by over/undershoots in the surface divergence $\beta$ associated with Marangoni forces counteracting strong up and downwellings. In S1 these over/undershoots result in large local $|\beta|$ which significantly contributes to the overall $\beta_{r m s}$. If the surface divergence model would be applicable in the above situation, we would expect to find a larger transfer velocity in S1 than in S0. In reality, however, this is not the case (cf. figure 9). A possible explanation for this could be the presence of high intensity small scale structures in the $\beta$ distribution of $\mathrm{S} 1$. These small spatial structures have small time scales for which the surface divergence model no longer provides accurate results as has been shown in Turney \& Banerjee (2013).

The validity of the surface divergence model for a shear-free surface boundary condition was shown by e.g. Magnaudet \& Calmet (2006); Herlina \& Wissink (2014); Wissink \& Herlina (2016). Attempts were also made to use the surface divergence model to determine the transfer velocity for cases with surface shear (e.g. Law \& Khoo 2002; Banerjee \& MacIntyre 2004; Turney 2016). Note that in the latter cases the effect of $S c$ was not evaluated. Figure $11 \mathrm{~d}$ shows the variation of $c_{\beta}$ with $M a / C a_{T}$. For the cases approaching free-slip, and the free-slip case itself, $c_{\beta}$ is found to be about 0.5 to 0.58 . The latter range of values is in good agreement with the LES results of Magnaudet \& Calmet (2006) and the clean surface experimental results of McKenna \& McGillis (2004). For $M a / C a_{T} \approx 5(\mathrm{~S} 2), c_{\beta}$ at $S c=32$ reduces to 0.35 . The results presented in figure $11 \mathrm{~d}$ suggest that the constant of proportionality in (1.3) needs to be reduced in order to deal with the inhibiting effects of surface contamination on the transfer velocity. A close examination of the results presented in figure 6 of McKenna \& McGillis (2004) indeed shows that each surface condition basically requires its own constant of proportionality. Values of $c_{\beta}<0.5$, such as in the experiments of Herlina \& Jirka (2008), might indicate a certain level of contamination or shear at the interface. Note that in the literature it is usually assumed that $k_{L} \propto S c^{-1 / 2}$. Later on in this paper evidence will be provided that this scaling is no longer valid in the presence of (instantaneous) surface shear as for instance induced by the presence of surfactants. Perhaps the usage of a different exponent for $S c$ could mitigate the need to adjust $c_{\beta}$ in (1.3), in which $D=1 /(\operatorname{Re} S c)$.

In agreement with figure 10, figure $11 \mathrm{~d}$ also shows that the data points obtained for different $S c$ only collapse for very small values of $M a / C a_{T}$, indicating again that the surface divergence model as given in (1.3) is only valid (in the absence of small time scales) for very low levels of contamination.

\subsubsection{Clean surface area}

The flow near the contaminated surface is affected by horizontal gradients in the surfactant concentration as can be seen in (2.7) and (2.8). These gradients generate Marangoni forces, of which the strength depends on $M a / C a_{T}$. The regions where the surfactant concentration is small are observed to reduce in size with increasing Marangoni forces as can be seen in the instantaneous visualisations of $\gamma$ shown in figure 12 .

In order to properly quantify the "surfactant-free" region we defined a threshold for the 


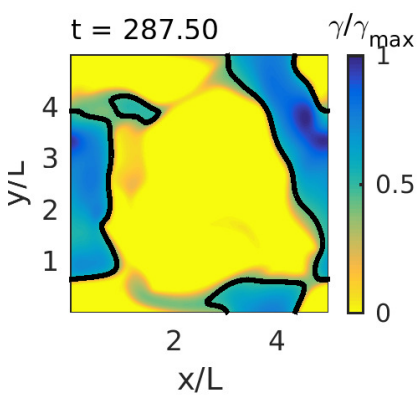

(a)

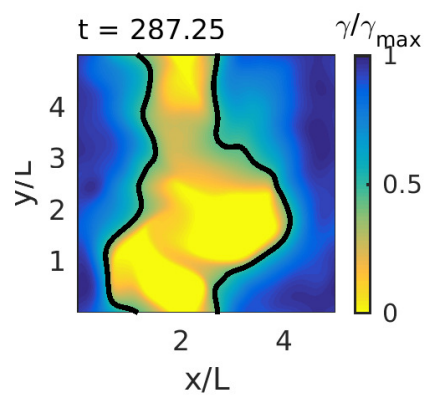

(b)

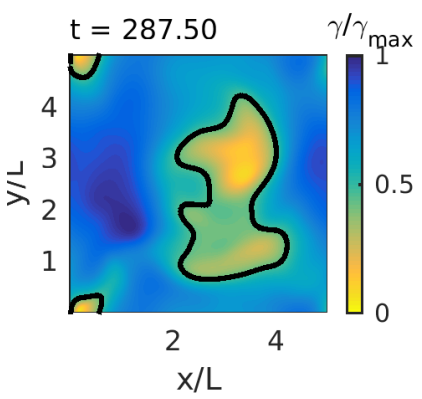

(c)

Figure 12. Effect of increasing $M a / C a_{T}$ on the "surfactant-free" fraction of the total surface area. a) S1, b) S2, c) S3. The solid black lines identify $\gamma / \gamma_{\max }=0.45$.

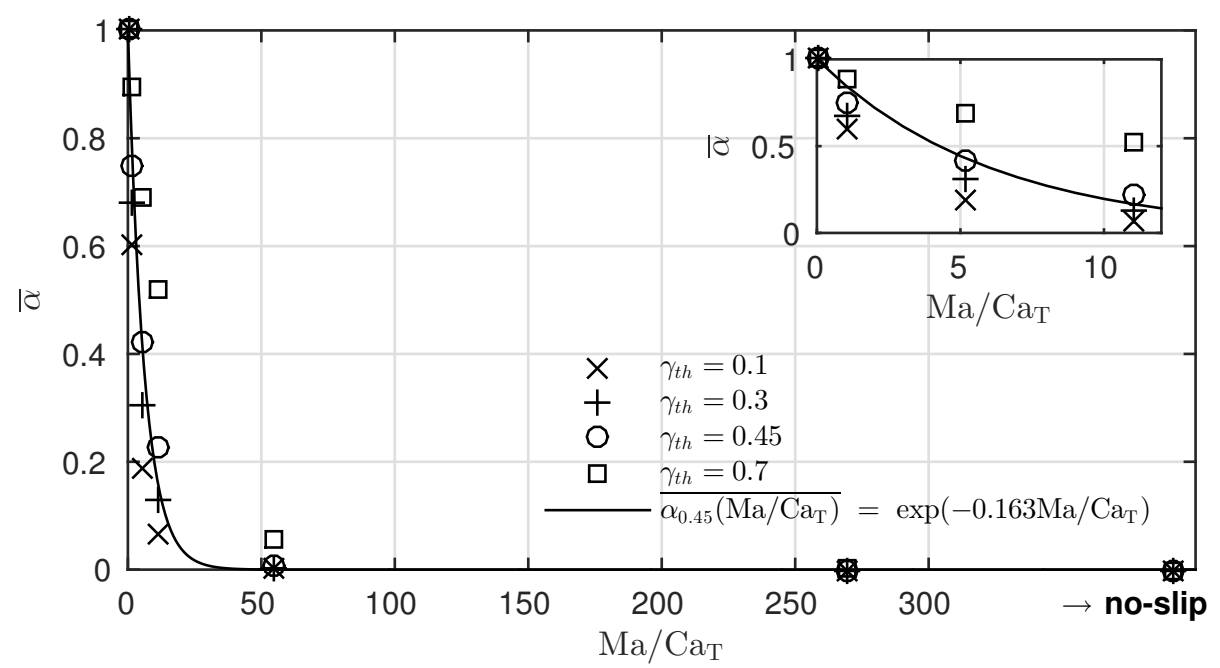

FIGURE 13. Variation of clean surface fraction $\bar{\alpha}$ with $M a / C a_{T}$

surfactant concentration, below which the local area is deemed to be clean. For this we normalised the surfactant concentration using the maximum instantaneous concentration $\gamma_{\max }$. The clean surface fraction $0 \leqslant \alpha \leqslant 1$ is subsequently defined by the fraction of the total area where

$$
\gamma / \gamma_{\max }<\gamma_{t h}
$$

Figure 12 also shows isolines of the threshold $\gamma_{t h}=0.45$. It will be shown below that this threshold serves as a very good measure for the time-averaged transfer velocity (cf. $\S 4.4)$. In S1 almost the entire surface is either virtually clean or very dirty. The clean and dirty parts are separated by a steep gradient in the surfactant concentration. Contrasting the results from $\mathrm{S} 1, \mathrm{~S} 2$ and $\mathrm{S} 3$, it can be seen that this gradient becomes more diffuse with increasing $\mathrm{Ma} / \mathrm{Ca}_{T}$, while simultaneously the clean surface fraction $\alpha$ gradually reduces in size.

Figure 13 shows the variation of the time-averaged clean surface fraction $\bar{\alpha}$ with $M a / C a_{T}$ when using thresholds $\gamma_{t h}=0.1,0.3,0.45$ and 0.7 . For $M a / C a_{T}<11$ a slight increase in contamination level is found to lead to a sharp reduction in $\bar{\alpha}$. Values of $M a / C a_{T} \geqslant 54$ are observed to correspond to severely contaminated surface conditions, 


\begin{tabular}{|c|c|c|c|c|c|c|c|}
\hline \multirow[t]{2}{*}{ Run } & \multirow[t]{2}{*}{$M a / C a_{T}$} & \multicolumn{6}{|c|}{$\rho\left(k_{L}, \alpha_{\gamma_{t h}}\right)$} \\
\hline & & $\gamma_{0.1}$ & $\gamma_{0.2}$ & $\gamma_{0.3}$ & $\gamma_{0.4}$ & $\gamma_{0.5}$ & $\gamma_{0.6}$ \\
\hline S1 & 1 & 0.7543 & 0.7391 & 0.6802 & 0.6060 & 0.5030 & 0.4151 \\
\hline S2 & 5 & 0.8466 & 0.7795 & 0.6864 & 0.5808 & 0.4707 & 0.4089 \\
\hline S3 & 11 & 0.9568 & 0.9497 & 0.9045 & 0.7738 & 0.4182 & 0.0696 \\
\hline
\end{tabular}

TABLE 2. Correlation coefficients at $S c=32$ for various thresholds.

where $\bar{\alpha} \approx 0$. Furthermore, the variation of $\bar{\alpha}$ with $M a / C a_{T}$ can be reasonably well represented by an exponential relationship. Denoting the clean surface fraction obtained using $\gamma_{t h}$ by $\overline{\alpha_{\gamma_{t h}}}$, the best fit for $\gamma_{t h}=0.45$ is found to be

$$
\overline{\alpha_{\gamma_{t h}}}=\overline{\alpha_{0.45}}=e^{-0.163 M a / C a_{T}} .
$$

Note that the parameter $M a / C a_{T}=R_{T} M a / W e_{T}$, which determines the strength of the Marangoni forces, is expected to be universal for this type of flow, where mass transfer is induced by isotropic turbulence diffusing from below. Hence, though not explicitly confirmed here, the above results are likely to be valid also for other values of $R_{T}$ than the ones considered here.

\subsection{Correlation between instantaneous $\alpha$ and instantaneous $k_{L}$}

Because of the free-slip and no-slip boundary conditions the instantaneous $\alpha$ is identical to unity in $\mathrm{S} 0$ and zero in SN, respectively. For $\gamma_{t h} \leqslant 0.5, \alpha$ is found to be virtually always zero in runs $\mathrm{S} 4\left(\mathrm{Ma} / \mathrm{Ca}_{T}=54\right)$ and $\mathrm{S} 5\left(\mathrm{Ma} / \mathrm{Ca}_{T}=269\right)$, indicating a severe contamination level. Hence, as in the no-slip case $\mathrm{SN}$, the instantaneous transfer velocity in S4 and S5 is likely to scale with $S c^{-2 / 3}$, which is in agreement with the scaling of the time averaged $K_{L}$ shown in figure 10. For cases S1, S2 and S3 the nonzero instantaneous clean surface fraction $\alpha$ is likely to affect $k_{L}$ significantly, such that increases in $k_{L}$ would be associated with increases in $\alpha$. This is confirmed for S2 in figure 14, which shows the evolution of $k_{L}$ and $\alpha_{\gamma_{t h}}$ for $\gamma_{t h}=0.1,0.3,0.5$ using $S c=32$. It can be seen that $k_{L}$ is strongly correlated with $\alpha_{\gamma_{t h}}$ for $\gamma_{t h}=0.1$ and that this correlation degrades when $\gamma_{t h}$ increases. This observation is quantified in table 2, showing the correlation coefficient $\rho\left(k_{L}, \alpha_{\gamma_{t h}}\right)$ for $\gamma_{t h}=0.1, \ldots, 0.6$ at $S c=32$. The table shows that (i) with increasing $\gamma_{t h}$ the degradation in correlation mentioned above increases significantly with increased level of contamination, and (ii) for $\gamma_{t h}<0.4$ the correlation improves with increasing $M a / C a_{T}$, while for larger $\gamma_{t h}$ it decreases.

Note that for the cases with $0<M a / C a_{T} \leqslant 11$ a maximum correlation is expected for a threshold $0<\gamma_{t h} \leqslant 1$ because

$$
\left.\begin{array}{ll}
\gamma_{t h}=0 & \Rightarrow \alpha \equiv 0 \\
\gamma_{t h}=1+\varepsilon & \Rightarrow \alpha \equiv 1
\end{array}\right\} \Rightarrow \rho\left(k_{L}, \alpha\right)=0,
$$

(where $\varepsilon>0$ is small). Further investigations have shown that for all cases in table 2 this maximum indeed exists and is reached for a threshold $0<\gamma_{t h}<0.2$. For such small $\gamma_{t h}$ the surfactant concentration within the identified clean area fraction $\alpha_{\gamma_{t h}}$ will be very low so that its local contribution to $k_{L}$ will be almost identical to the free-slip case $\left(M a / C a_{T}=0\right)$. As can be seen in figure 9 , assuming a fifty-fifty split between perfectly clean and extremely dirty areas, the clean area's contribution to the overall $K_{L}$ at $S c=32$ will be 2.75 times larger than the dirty area's contribution.

Additionally to the time evolution of $\alpha$ and $k_{L}$ - obtained using (4.3) - figure 14 

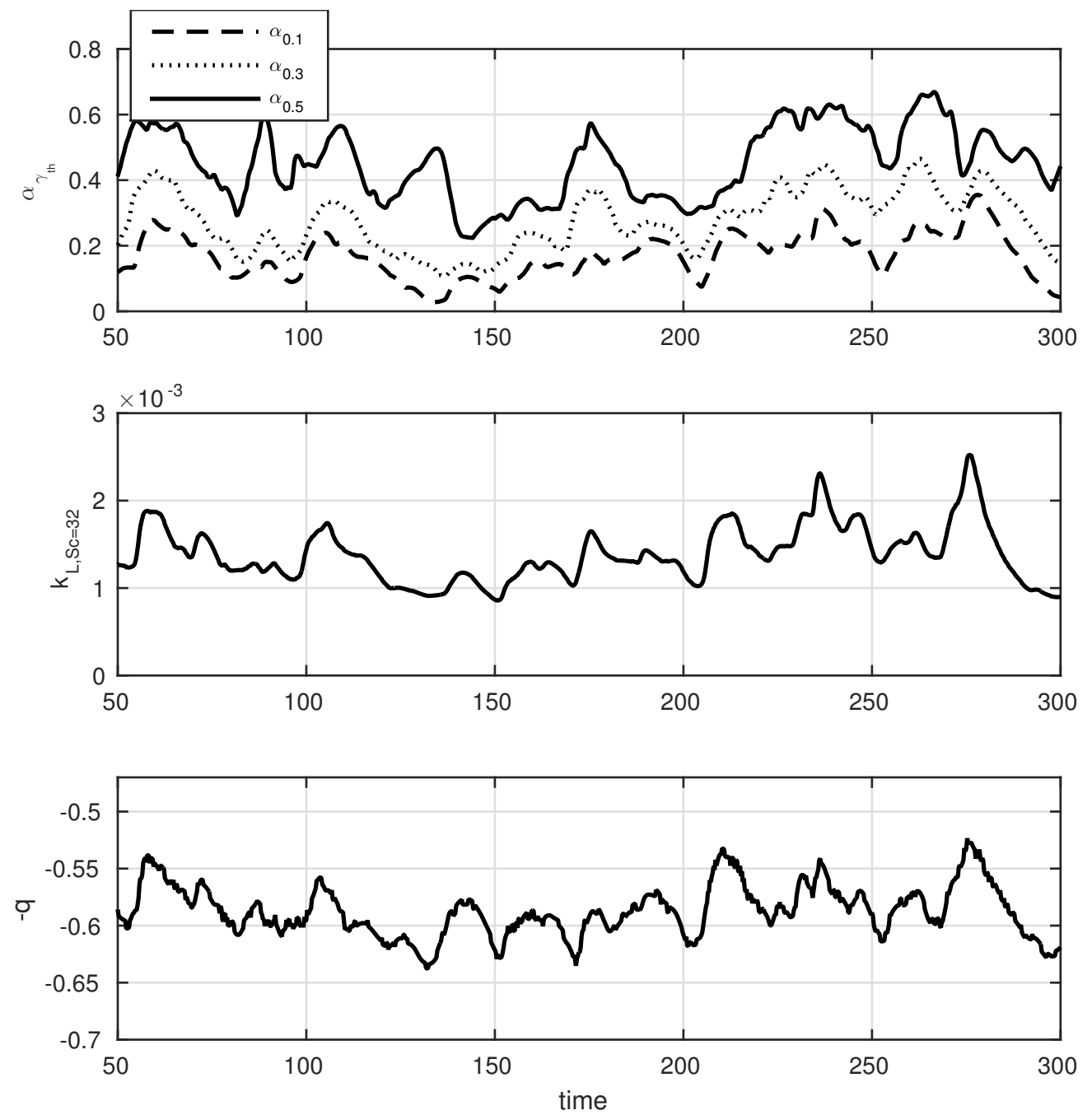

FiguRE 14. Time series of clean surface fraction $\alpha$ (upper pane), the corresponding $k_{L}$ (middle pane) and $-q$ (lower pane) evaluated using the horizontally-averaged instantaneous concentration profile from run $\mathrm{S} 2$.

(lower pane) also shows the evolution of the exponent $-q$ in the instantaneous power law dependency $k_{L} \propto S c^{-q}$. In each $M a / C a_{T}$ case, transport equations for five different $S c$ were solved simultaneously - i.e. with exactly the same turbulent flow-field - allowing an unbiased determination of the instantaneous exponent $-q$ using the least squares method. By comparing the temporal evolution of $-q$ and $\alpha$, it can be seen that when $\alpha$ reduces $q$ becomes larger and moves in the direction of $2 / 3$, which is valid for severe contamination levels. Also, when $\alpha$ increases, $q$ decreases and moves towards $1 / 2$, which is valid for clean surface conditions.

The above observations support the idea presented in $\S 3$ of estimating the mean $K_{L}$ using the average clean surface fraction $\bar{\alpha}$. 
4.4. Comparison between observed mean $q, c$ and $K_{L}$ with their predictions based on $\bar{\alpha}$

In $\S 4.3$ a good temporal correlation between $\alpha_{0.1}$ and $k_{L}$ for S1, S2, S3 was obtained. This does not necessarily imply that by using $\gamma_{t h}=0.1$ to determine $\bar{\alpha}$, the prediction of the magnitude of the normalised transfer velocity

$$
\frac{K_{L}}{u_{\infty} R_{T}^{-1 / 2}}(\bar{\alpha})=c(\bar{\alpha}) S c^{-q(\bar{\alpha})},
$$

where $c=c(\bar{\alpha})$ and $q=q(\bar{\alpha})$ are determined as in (3.5) to (3.7), is correct. This will be further investigated below.

From our results it is possible to directly determine $q$ and $c$ for each $M a / C a_{T}$ case using the least squares method. In figure 10 it is observed that $q$ - corresponding to the gradients of the lines in the log-log plot - varies with $M a / C a_{T}$. This relation is investigated further in figure $15 \mathrm{a}$, which shows an overall smooth variation of $-q$ from $-1 / 2$ to $-2 / 3$ with increasing $M a / C a_{T}$. Similarly, as seen in figure $15 \mathrm{~b}$, the constant of proportionality $c$ is also found to smoothly vary between its free-slip value $c_{f}$ and its no-slip value $c_{n}$.

Comparing the results shown in figures $9 \mathrm{a}, 13$ and 15, a strong correlation can be observed between $\bar{\alpha}$ and each of the time-averaged values $q, c$ and $K_{L}$. This indicates that in our simulations (where $R_{T}$ and $u_{\infty}$ are approximately the same) $K_{L}=c S c^{-q}$ can be modelled as a function of $\bar{\alpha}$ alone.

To determine the surfactant-free fraction $\bar{\alpha}$ in (3.5) and (3.7) from the numerical results, we need to determine the optimum threshold $\gamma_{t h}$ in (4.4) so that the sum of the squared errors

$$
\operatorname{SSE}\left(\overline{\alpha_{\gamma_{t h}}}\right)=\sum_{S 0}^{S N}\left[K_{L}-K_{L}\left(\overline{\alpha_{\gamma_{t h}}}\right)\right]^{2}
$$

is minimum, where $K_{L}$ is the reference value directly obtained from the numerical results and $K_{L}\left(\overline{\alpha_{\gamma_{t h}}}\right)$ is the predicted transfer velocity calculated using (3.5), (3.7) and (1.6). The parameters $c_{f}=1.55$ (1.6 in Herlina \& Wissink 2014) and $c_{n}=0.94$ (0.95 in Herlina \& Wissink 2016) are determined from the results of runs S0 (free-slip) and SN (no-slip), respectively. Note that $c_{f}$ and $c_{n}$ may vary depending on the location where $u_{\infty}$ and $L_{\infty}$ are evaluated (see also $\S 4.1$ ). Since our simulations fall into the low to moderate turbulence intensity regime, we assume that $r=1 / 2$ in (1.6).

As shown in figure 16 the $S S E$ obtained using $\gamma_{t h}=0.1, \ldots, 0.5$ reaches a minimum between $\gamma_{t h}=0.4$ and 0.5. Despite the good temporal correlations between $\alpha$ and $k_{L}$ obtained for smaller $\gamma_{t h}$, figure 16 shows that by using such small $\gamma_{t h}$ to determine $\bar{\alpha}$ the actual magnitude of $K_{L}=\overline{k_{L}}$ is significantly underestimated. Unless specified differently, a threshold of $\gamma_{t h}=0.45$ was used for the determination of $\bar{\alpha}$ below.

To test the quality of our prediction of $q$ and $c$ using $\overline{\alpha_{0.45}}$, in figure 15 the predicted data points are shown alongside the numerical results, showing a reasonable agreement. In addition, the dashed line, which was produced using the exponential relationship (4.5) between $M a / C a_{T}$ and $\overline{\alpha_{0.45}}$, can be seen to provide a nice interpolation between the data points.

Finally, based on the reasonably good prediction achieved above for $q$ and $c$, it is expected that the proposed model based on the clean surface fraction also provides a prediction of at least similar quality for the transfer velocity. This is indeed confirmed in figure 17, illustrating that the calculation of the normalised transfer velocity from (4.7) both (i) directly by employing the observed $\overline{\alpha_{0.45}}$

$$
\frac{K_{L}}{u_{\infty} R_{T}^{-1 / 2}}\left(\overline{\alpha_{0.45}}\right)
$$




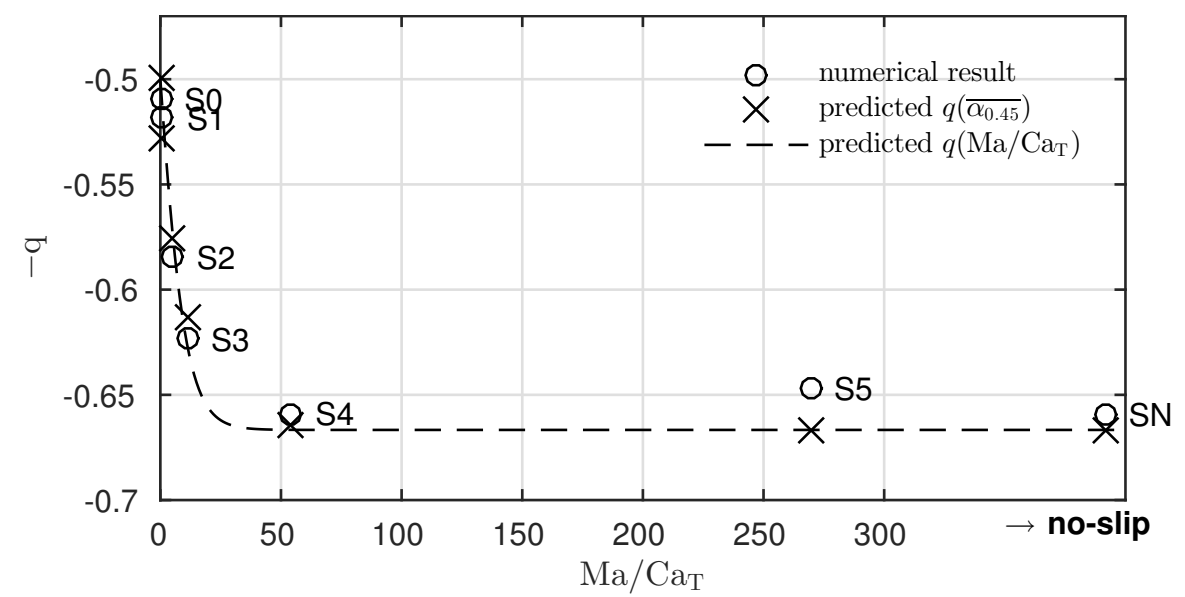

(a)

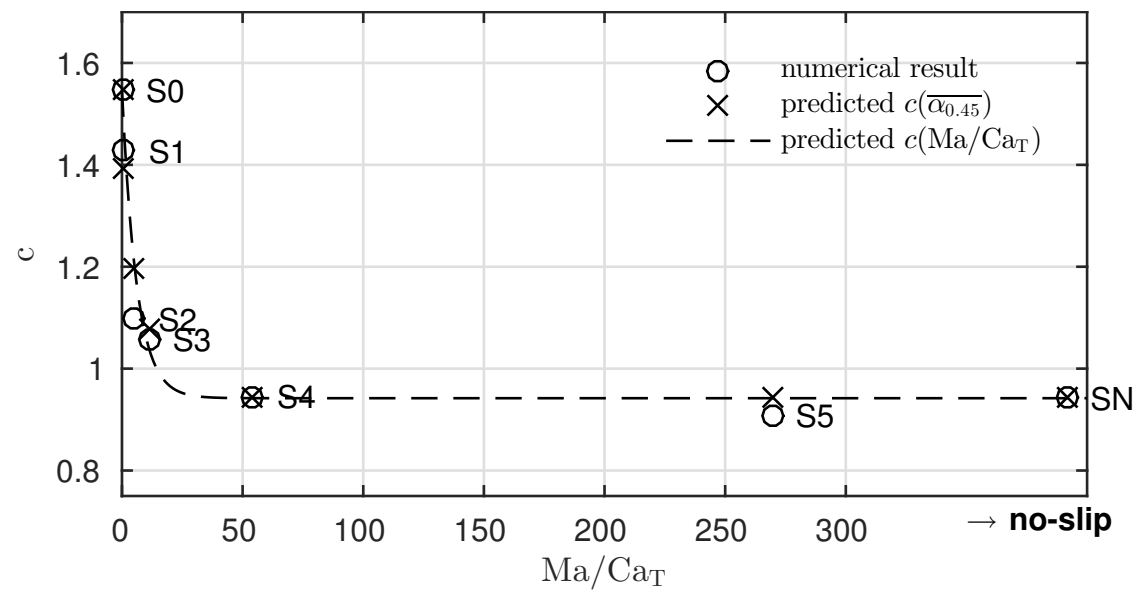

(b)

Figure 15. Variation of a) exponent $-q$ and b) constant $c$ with $M a / C a_{T}$. Note results are averaged from $t=150-300$ and the least-squares method was used to extract $-q$ and $c$ from the numerical results.

and (ii) indirectly by using (4.5) where $\overline{\alpha_{0.45}}$ is estimated as a function of $M a / C a_{T}$

$$
\frac{K_{L}}{u_{\infty} R_{T}^{-1 / 2}}\left(M a / C a_{T}\right)=\frac{K_{L}}{u_{\infty} R_{T}^{-1 / 2}}\left(\overline{\alpha_{0.45}}\left(M a / C a_{T}\right)\right) .
$$

agree well with the normalised transfer velocity $K_{L}$ that was directly obtained from the numerical data. Especially, the initially steep decline in $K_{L}$ for small $M a / C a_{T}$ is well captured with a maximum error of $8 \%$. Also, for larger $M a / C a_{T}$ a smooth transition to severely contaminated conditions is predicted.

\section{Conclusions}

In order to study the influence of surfactants on interfacial mass transfer, direct numerical simulations (DNS) with various levels of contamination have been performed. At the surface of the computational domain the waves are assumed to be very shallow so 


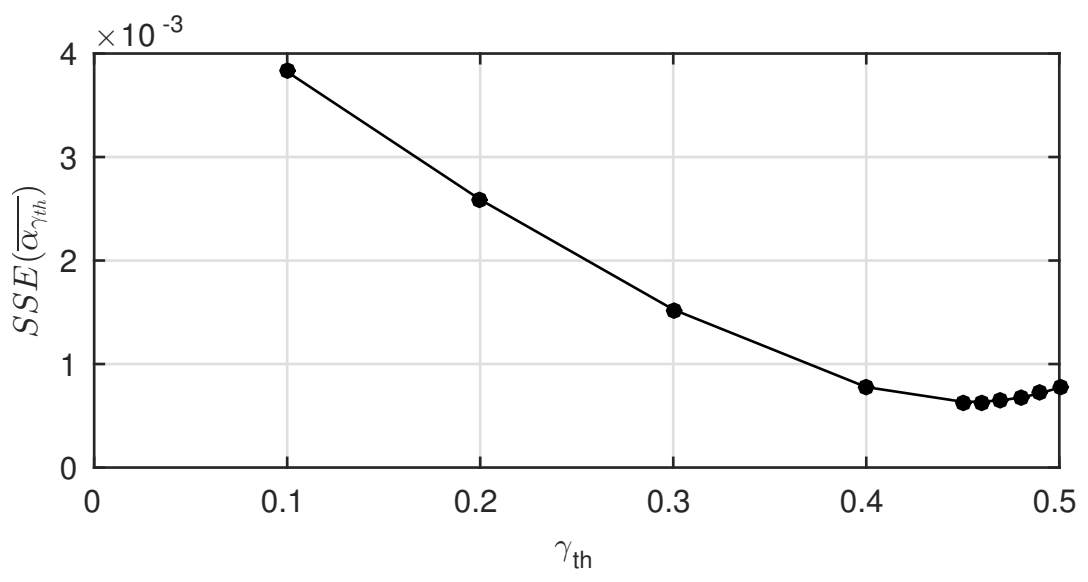

FIGURE 16. Sum of squared errors defined in (4.8).

that a rigid lid assumption can be employed. In all simulations, mass transfer - promoted by introducing isotropic turbulence at the bottom of the computational domain - is calculated simultaneously for five different Schmidt numbers, $S c=2,4,8,16,32$. At the bottom of all simulations the same turbulent flow field is introduced. Consequently, both the turbulent Reynolds numbers and integral length scales are kept within a small range of $R_{T}=129 \pm 12$ and $L_{\infty}=0.96 \pm 0.08$, which allows for the first time an unbiased study of the effect of $M a / C a_{T}$ on $S c$. In the surface plane, an advection-diffusion equation is solved for the surfactant concentration. The model described in Shen et al. (2004) is adopted to account for the influence of gradients in the surfactant concentration on the near surface turbulent flow field, which in turn affects the interfacial mass transfer. The ratio of the Marangoni number and the turbulent capillary number, $M a / C a_{T}$, is used as a measure of contamination.

In our previous simulations we investigated interfacial mass transfer for a range of $R_{T}$ and $S c$ up to 500 using either a free-slip boundary condition at the surface (Herlina \& Wissink 2014) or a no-slip boundary condition (Herlina \& Wissink 2016). The power dependency of $K_{L}$ on $R_{T}$ was found to be independent on the surface boundary conditions. For the range of $R_{T}$ considered, it was shown that $K_{L} \propto R_{T}^{-1 / 2}$ for $R_{T}<R_{T, \text { crit }}$ and $K_{L} \propto R_{T}^{-1 / 4}$ for $R_{T}>R_{T, \text { crit }}$, where $R_{T, \text { crit }} \approx 500$. This suggests that the same power dependency of $K_{L}$ on $R_{T}$ is also likely to be valid for any level of contamination. Hence, as in the present simulations $R_{T}<R_{T, \text { crit }}$, the transfer velocity is normalised using $R_{T}^{-1 / 2} u_{\infty}$. On the other hand, for the entire range of $S c$ investigated in our previous simulations it was confirmed that the transfer velocity scales with $S c^{-1 / 2}$ and $S c^{-2 / 3}$ for the free-slip and no-slip cases, respectively.

In the present DNS, it is found that even small levels of contamination significantly affect the near surface turbulence so that $K_{L}$ no longer scales with $S c^{-1 / 2}$. In the no-slip case the surface divergence is strictly zero, which significantly reduces the interchange of saturated and unsaturated fluid. Because of the nearly zero surface divergence, a similar reduction in gas exchange can also be expected in cases with large $M a / C a_{T}$. So even though the near surface hydrodynamics of highly contaminated surfaces $\left(\mathrm{Ma} / \mathrm{Ca} a_{T} \rightarrow \infty\right)$ does not entirely converge to the no-slip case, the scaling of the transfer velocity becomes similar, i.e. $K_{L} \propto S c^{-2 / 3}$. For intermediate levels of contamination, $K_{L}$ is found to approximately scale with $S c^{-q}$, where $q$ is shown to gradually increase from $1 / 2$ to $2 / 3$ with increasing levels of contamination. Consequently, the surface divergence model, 


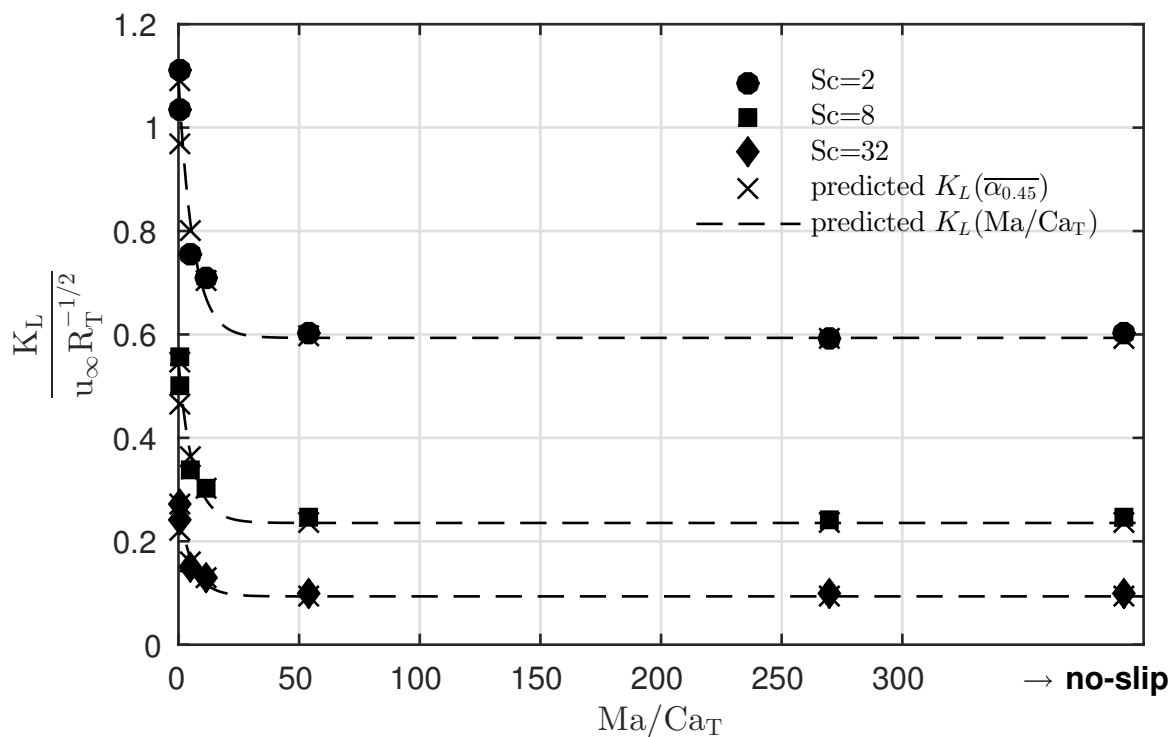

(a)

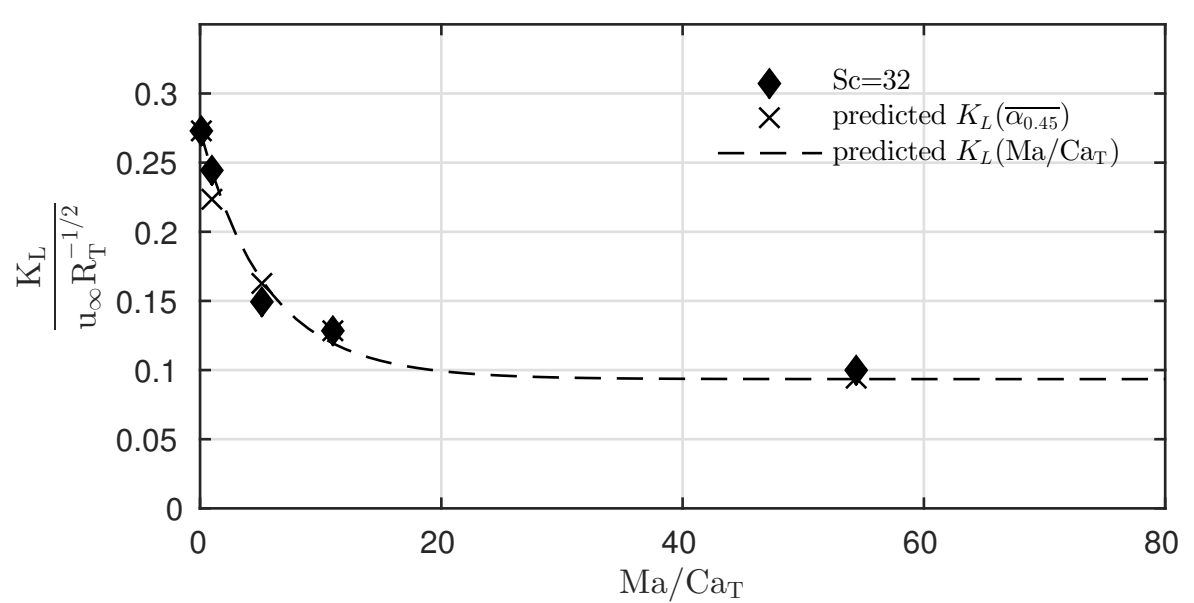

(b)

Figure 17. Comparison of the predicted $K_{L}$ with present numerical results as a function of $M a / C a_{T}$. a) Results obtained for $S c=2,8,32$, b) Close-up of $S c=32$ results for low to moderate $\mathrm{Ma} / \mathrm{Ca} a_{T}$.

which assumes that $K_{L} \propto S c^{-1 / 2}$, is no longer accurate in the presence of even slight contamination.

It is shown that Marangoni forces generate instantaneous shear at the surface which for small to moderate $M a / C a_{T}$ tends to increase with contamination level. For large $\mathrm{Ma} / \mathrm{Ca}$ the surface flow-field becomes virtually divergence-free, even though, compared to the no-slip boundary condition, a non-zero 2D flow-field persists.

For small $\mathrm{Ma} / \mathrm{Ca} \mathrm{a}_{T}$, regions with very low surfactant concentration are found to appear, which are observed to gradually reduce in size with increasing $M a / C a_{T}$. The fraction of the surface that these clean regions occupy is represented by the parameter $0 \leqslant \alpha \leqslant 1$. For constant $S c$ and $R_{T}$ the transfer velocity is found to correlate very well with the clean surface fraction, suggesting that $K_{L}$ can be regarded as a function of $R_{T}, S c$ and $\alpha$. By 
assuming that the "clean" regions behave as a free-slip boundary, while the remaining "dirty" regions behave as a no-slip boundary, a model for the transfer velocity that accounts for the presence of surfactants is obtained:

$$
K_{L}=c u_{\infty} S c^{-q} R_{T}^{-r},
$$

with

$$
\begin{gathered}
c=\bar{\alpha} c_{f}+(1-\bar{\alpha}) c_{n}, \\
q=\frac{2}{3}-\frac{\bar{\alpha} c_{f}}{6 c},
\end{gathered}
$$

where $c_{f}$ and $c_{n}$ are the constants of proportionality for the free-slip and no-slip cases, respectively, and $r=1 / 2$ or $1 / 4$ depending on the turbulence intensity. A reasonably good prediction of the average transfer velocity, with a maximum error of $8 \%$, is obtained by identifying $\alpha$ with the average surface fraction where $\gamma / \gamma_{\max }<0.45$.

The present results confirm that to predict mass transfer accurately, surface contamination effects need to be taken into account. Hence, information is needed of both the turbulent flow characteristics and the concentration distribution of the surfactant. The latter may become possible with further advances in remote sensing technology, which will allow a quantification of the mean clean surface fraction $\bar{\alpha}$.

\section{Acknowledgements}

This research was funded by the German Research Foundation (DFG UH242/6-1). The authors would like to thank the Steinbuch Centre for Computing (SCC) in Karlsruhe for providing computing time on the HP XC 3000. We would also like to thank the anonymous reviewers for their invaluable suggestions for improvement.

\section{REFERENCES}

Asher, W. E. \& PAnkow, J.F. 1986 The interaction of mechanically generated turbulence and interfacial films with a liquid phase controlled gas/liquid transport process. Tellus 38B, 305-318.

BAnerjee, S. \& MacIntyre, S 2004 The air-water interface: turbulence and scalar exchange. In Advances in Coastal and Ocean Engineering, pp. 181-237. World Scientific.

Banerjee, S., Scott, D. S. \& Rhodes, E. 1968 Mass transfer to falling wavy liquid films in turbulent flow. Ind. Eng. Chem. Fundam. 7 (1), 22-27.

Brumley, B. H. \& JiRkA, G. H. 1987 Near-surface turbulence in a grid-stirred tank. J. Fluid Mech. 183, 235-263.

Calmet, I. \& Magnaudet, J. 2003 Statistical structure of high-reynolds-number turbulence close to the free surface of an open-channel flow. J. Fluid Mech. 474, 355-378.

CoAntic, M. 1986 A model of gas transfer across air-water interfaces with capillary waves. $J$. Geophys Res-Oceans 91 (C3), 3925-3943.

DANCKWERTs, P. V. 1951 Significance of liquid-film coefficients in gas absorption. Ind. Engng Chem. 43 (6), 1460-1467.

Davies, J. T. 1972 Turbulence Phenomena. Academic Press, N.Y.

Espedal, H. A., Johannessen, O. M. \& Knulst, J. 1996 Satellite detection of natural films on the ocean surface. Geophys. Res. Letters 23 (22), 3151-3154.

Fortescue, G. E. \& Pearson, J. R. 1967 On gas absorption into a turbulent liquid. Chem. Engng Science 22 (9), 1163-1176.

Handler, R.A, Leighton, R.I, Smith, G.B \& Nagaosa, R. 2003 Surfactant effects on passive scalar transport in a fully developed turbulent flow. Int. J. Heat Mass Tran. 46 (12), 22192238 . 
Hasegawa, Y. \& Kasagi, N. 2008 Systematic analysis of high Schmidt number turbulent mass transfer across clean, contaminated and solid interfaces. Int. J. Heat Fluid Flow 29 (3), $765-773$.

Herlina \& JiRKA, G. H. 2008 Experiments on gas transfer at the air-water interface induced by oscillating grid turbulence. J. Fluid Mech. 594, 183-208.

Herlina, H. \& Wissink, J.G. 2014 Direct numerical simulation of turbulent scalar transport across a flat surface. J. Fluid Mech. 744, 217-249.

Herlina, H. \& Wissink, J.G. 2016 Isotropic-turbulence-induced mass transfer across severely contaminated water surface. J. Fluid Mech. 797, 665-682.

Hopfinger, EJ \& Toly, J-A 1976 Spatially decaying turbulence and its relation to mixing across density interfaces. J. Fluid Mech. 78 (1), 155-175.

JÄhne, B. \& Haussecker, H. 1998 Air-water gas exchange. Annu. Rev. Fluid Mech. 30, 443468.

JeOng, J. \& Hussain, F. 1995 On the identification of a vortex. J. Fluid Mech. 285, 69-94.

Kermani, A., Khakpour, H.R., Shen, L. \& Igusa, T. 2011 Statistics of surface renewal of passive scalars in free-surface turbulence. J. Fluid Mech. 678, 379-416.

Khakpour, H.R., Shen, L. \& Yue, D.K.P. 2011 Transport of passive scalar in turbulent shear flow under a clean or surfactant-contaminated free surface. J. Fluid Mech. 670, 527-557.

Kubrak, B., Herlina, H., Greve, F. \& Wissink, J.G. 2013 Low-diffusivity scalar transport using a WENO scheme and dual meshing. J. Comput. Phys. 240, 158-173.

Lamont, J. C. \& ScotT, D. S. 1970 An eddy cell model of mass transfer into surface of a turbulent liquid. AIChE J. 16 (4), 513-519.

Law, C. N. S. \& Kноо, B. C. 2002 Transport across a turbulent air-water interface. AIChE J. 48 (9), 1856-1868.

LEDWELL, J. J. 1984 The variation of the gas transfer coefficient with molecular diffusity. In Gas transfer at water surfaces, pp. 293-302. Springer.

Liu, X., Osher, S. \& Chan, T. 1994 Weighted essentially non-oscillatory schemes. J. Comput. Phys. 115 (1), 200-212.

Magnaudet, J. \& CALmet, I. 2006 Turbulent mass transfer through a flat shear-free surface. J. Fluid Mech. 553, 155-185.

McCready, M.J., Vassiliadou, E. \& Hanratty, T.J. 1986 Computer-simulation of turbulent mass-transfer at a mobile interface. AIChE J. 32 (7), 1108-1115.

McKenna, S. P. \& McGillis, W.R. 2004 The role of free-surface turbulence and surfactants in air-water gas transfer. Int. J. Heat Mass Tran. 47 (3), 539-553.

NA, Y. \& HANRATTY, T. J. 2000 Limiting behavior of turbulent scalar transport close to a wall. International Journal of Heat and Mass Transfer 43 (10), 1749 - 1758.

Nagaosa, R. \& HandleR, R.A. 2003 Statistical analysis of coherent vortices near a free surface in a fully developed turbulence. Phys. Fluids 15 (2), 375-394.

Notter, R. H. \& Sleicher, C. A. 1971 The eddy diffusivity in the turbulent boundary layer near a wall. Chem. Eng. Sci. 26 (1), 161-171.

Peirson, W. L. \& Banner, M. L. 2003 Aqueous surface layer flows induced by microscale breaking wind waves. J. Fluid Mech. 479, 1-38.

Perot, B. \& Moin, P. 1995 Shear-free turbulent boundary layers. Part 1. Physical insights into near-wall turbulence. J. Fluid Mech. 295, 199-227.

Salter, M.E., Upstill-Goddard, R.C., Nightingale, P.D., Archer, S.D., Blomquist, B., Ho, D.T., Huebert, B., Schlosser, P. \& YAng, M. 2011 Impact of an artificial surfactant release on air-sea gas fluxes during Deep Ocean Gas Exchange Experiment II. J. Geophys. Res: Oceans 116 (C11).

Shen, L., Yue, D.K.P. \& Triantafyllou, G.S. 2004 Effect of surfactants on free-surface turbulent flows. J. Fluid Mech. 506, 79-115.

Theofanous, T. G., Houze, R. N. \& Brumfield, L. K. 1976 Turbulent mass transfer at free, gas liquid interfaces with applications to open channel, bubble and jet flows. Int. J. Heat Mass Tran. 19 (6).

TsaI, W.-T. 1996 Impact of a surfactant on a turbulent shear layer under the air-sea interface. J. Geophys. Res. Oceans 101 (C12), 28557-28568.

Turney, D. E. 2016 Coherent motions and time scales that control heat and mass transfer at wind-swept water surfaces. J. Geophys. Res. Oceans 121 (12), 8731-8748. 
Turney, D. E. \& BAnerJee, S. 2013 Air-water gas transfer and near-surface motions. J. Fluid Mech. 733, 588-624.

Turney, D. E., Smith, W. C. \& Banerjee, S. 2005 A measure of near-surface fluid motions that predicts air-water gas transfer in a wide range of conditions. Geophys. Res. Lett. 32, L04607.

Walker, D. T., Leighton, R. I. \& Garza-Rios, L. O. 1996 Shear-free turbulence near a flat free surface. J. Fluid Mech. 320, 19-51.

Wissink, J.G. 2004 On unconditional conservation of kinetic energy by finite-difference discretisations of the linear and non-linear convection equation. Comput. Fluids 33, 315-343.

Wissink, J. G. \& Herlina, H. 2016 Direct numerical simulation of gas transfer across the air-water interface driven by buoyantconvection. J. Fluid Mech. 787, 508-540. 